\title{
The Coulomb Gas at Low Temperature and Low Density*
}

\author{
Joseph G. Conlon ${ }^{1}$, Elliott H. Lieb ${ }^{2}$, and Horng-Tzer Yau ${ }^{3}$ \\ ${ }^{1}$ Department of Mathematics, University of Missouri, Columbia, MO 65211, USA \\ ${ }^{2}$ Departments of Mathematics and Physics, Princeton University P.O.B. 708, Princeton, \\ NJ 08544, USA \\ ${ }^{3}$ Courant Institute, New York University, 251 Mercer Street, New York, NY 10012, USA
}

\section{Dedicated to Roland Dobrushin}

\begin{abstract}
We study the quantum Coulomb Gas of $N$ particles with Hamiltonian $H$ at low temperature and negative values of the chemical potential $\mu$. If $\mu$ is sufficiently negative the Coulomb gas is approximately a perfect rare gas of charged particles, as expected. The interesting fact is that for higher (but still negative) values of $\mu$ the gas changes to a rare gas of some atom or molecule (which is most likely neutral). The type of molecule is determined by the ground state of the Hamiltonian $H-\mu N$ with center of mass motion removed.
\end{abstract}

\section{Introduction}

In this paper we are concerned with the thermodynamic properties of a quantum mechanical Coulomb gas of nuclei and electrons at very low temperature and density and to validate certain predictions of the Saha equation [10, 12], which is the equation that governs the regime. (Actually, Saha was interested in the solar chromosphere where the temperature is high by earthly standards and the pressure is low, but this equation extends to the regime of very low temperature and even lower density that we are considering here.)

Consider a system composed of $S$ species of charged particles (electrons and various nuclei) placed in a large box $\Lambda$ of volume $|\Lambda|$. If $N_{i}$ is the number of particles of species $i$, then $\varrho_{i}=N_{i} /|\Lambda|$ is its density. If the $\varrho_{i}$ 's and $\beta=\left(k_{B} T\right)^{-1}$ (with $k_{B}=$ Boltzmann's constant and $T=$ temperature) are fixed and $\Lambda \rightarrow \infty$ in a suitable way, we expect that, the long-range nature of the Coulomb potential notwithstanding, the intensive quantities such as pressure, free energy per unit volume, etc. have well defined limits and that these limits should have the correct convexity

\footnotetext{
* Work partially supported by U.S. National Science Foundation grant DMS 8600748 (J.C.),
} PHY 85-15288-(A03) (E.L.) and DMS-8601978 and DMS-8806731 (H.-T.Y.) 
properties with respect to the $\varrho_{i}$ 's and $T$. This, indeed, was shown to be true by Lieb and Lebowitz [8]. They also proved the following facts:

1. The thermodynamic properties as calculated in the microcanonical (fixed energy), canonical (fixed $N_{i}$ ) and grand canonical (fixed chemical potentials $\mu_{i}$ ) all agree with each other.

2. For the grand canonical ensemble, with partition function $\Xi$, the value of the pressure

$$
p=|\Lambda|^{-1} \beta^{-1} \ln \Xi
$$

is the same as that obtained from $\Xi^{0}$, which is the value of $\Xi$ in which the summation over all states is replaced by the summation over the states in which the total charge of the particles is zero. [Note: It is a fact that $\Xi \gg \Xi^{0}$, but nevertheless $|\Lambda|^{-1}\left(\ln \Xi-\ln \Xi^{0}\right) \rightarrow 0$ as $\Lambda \rightarrow \infty$.] In this paper we shall work always with the grand canonical ensemble and we let

$$
\boldsymbol{\mu}=\left(\mu_{1}, \ldots, \mu_{S}\right) \text { and } \mathbf{N}=\left(N_{1}, \ldots, N_{S}\right)
$$

denote the chemical potentials and particle numbers.

Now let us inquire about the behavior of the system when $\varrho=\sum_{i} \varrho_{i}$ is very small. Lebowitz and Pena [7] proved that when $T$ is fixed the free energy per unit volume becomes $\beta^{-1} \sum_{i} \varrho_{i} \ln \varrho_{i}$ in this limit. In other words, the system becomes a (neutral) mixture of ideal gases formed by the individual particles. This can also be called a plasma. The result is not surprising; at low density the entropic contribution to the free energy, $k_{B} T \varrho \ln \varrho$ dominates all binding energies, and any nontrivial bound complex "evaporates."

The situation is more interesting if we let $T \rightarrow 0$ as we let the $\varrho_{i}$ 's $\rightarrow 0$. (In fact we let $\varrho \approx e^{-\beta}$.) In this case, as $T$ and $\varrho$ go to zero the properties of the system are governed by the Saha equation $[10,12]$ which predicts that one will have either an ideal gas of the elementary particles as before or else an ideal gas of some bound complex. The entropy does not necessarily win.

To illustrate this phenomenon suppose there are carbon nuclei, oxygen nuclei, and electrons and let $E_{\mathrm{CO}_{2}}$ and $\mathrm{N}_{\mathrm{CO}_{2}}$ denote the ground state energy and particle number of one $\mathrm{CO}_{2}$ molecule. The relevant energy for calculating $\Xi$ is $E(\mathbf{N})-\mu \cdot \mathbf{N}$, where $E(\mathbf{N})$ is the energy of some state of the system with particle numbers $\mathbf{N}$. Presumably it is possible to choose $\mu$ so that two things are true:

1. $\mathbf{e} \equiv E_{\mathrm{CO}_{2}}-\boldsymbol{\mu} \cdot \mathbf{N}_{\mathrm{CO}_{2}}>0$.

2. $E(\mathbf{N})-\boldsymbol{\mu} \cdot \mathbf{N}>\mathbf{e}+c$ for all other states, except for the vacuum, $\mathbf{N}=0$. Here $c>0$ is some fixed constant.

While it lies beyond present rigorous technology to prove that such a $\boldsymbol{\mu}$ exists, one probably does. If not there is surely some other neutral molecule or atom (or a neutral pair of charged complexes) that replaces $\mathrm{CO}_{2}$ and for which (1) and (2) above are true. Let us here assume (1) and (2) for $\mathrm{CO}_{2}$ and continue to compute

$$
\Xi=\sum_{\mathbf{N}} \sum_{\text {states }} \exp [-\beta(E(\mathbf{N})-\boldsymbol{\mu} \cdot \mathbf{N})] .
$$

We decompose $\Lambda$ into cells of very large but fixed size with volume of the order $\exp [\beta \varepsilon]$. Let us pretend that the interaction between cells can be ignored 
(validating this is difficult and is the primary concern of this paper). The leading term in $\Xi$ (for a cell) comes from the vacuum, $\mathbf{N}=0$. The next most important term is $\exp [-\beta \varepsilon]$. What about the excited states of $\mathrm{CO}_{2}$ and the states for other mixtures of particles? These, it turns out, are small when $\beta$ is very large (validating this is the second main concern of this paper). The entropy, $S$, enters in the combination $T S$ and thus can be controlled when $T$ is small. The energy terms other than $E_{\mathrm{CO}_{2}}$ are controlled by the constant $c>0$ in (2) above. In short, any cell is probably empty (the vacuum) but when it does contain some particles they are in the form of a single $\mathrm{CO}_{2}$ molecule.

Results as described above (and with the same assumptions) were proved by Fefferman [5] for the case of protons and electrons. Then the hydrogen atom replaces the $\mathrm{CO}_{2}$ atom. The new features of our paper can be summarized as follows.

1. Our method is completely different. It exploits the localization formula (i.e. the restriction of particles to cells) introduced in our proof [2] of the $N^{7 / 5}$ law for bosons. In the present context we regard it as simpler than Fefferman's method.

2. The corrections to the simple Saha formula (i.e. the above picture of an ideal gas of $\mathrm{CO}_{2}$ molecules) should, on physical grounds, be of the order $\exp (-\beta \varepsilon)$ and not $1 / \beta$ as Fefferman obtains. Our method is able to accomplish this. Moreover, as $\mu$ is changed one passes from one phase to another (i.e. from a plasma to $\mathrm{CO}_{2}$ atoms to some other complex, etc.). In the limit $T \rightarrow 0$ the transition is sharp; when $T \neq 0$ the width (in $\mu$ space) should be of the order $\exp (-\beta \varepsilon)$, not $1 / \beta$. Again, we are able to show this.

3. We handle an arbitrary mixture of particles - not merely electrons and protons.

4. We are able to bound a large class of observables by one simple formalism using the Feynman-Hellman theorem; cf. (6.9)-(6.13). Again, our error terms are of order $e^{-\varepsilon \beta}$.

In the main part of this paper we shall concentrate primarily on estimates of the pressure and density. Our resulrs about expectation values of observables are standard consequences of convexity (i.e. the "Feynman-Hellman theorem") and therefore we present them only briefly in the last section.

\section{Main Results}

The Hamiltonian to be considered is the quantum Coulomb Hamiltonian $H$ acting on a Fock space of charged particles. The definition of Fock space will be given later. It is important, however, to understand the Hamiltonian $H$ on the subspace of fixed particle numbers. We shall assume in this paper that there is only a finite number of different species of particles (various nuclei and electrons) and shall denote this number by $S$. Let $x=\left(x_{1}, \ldots, x_{N}\right)$ denote the coordinates of $N$ particles in $\mathbf{R}^{3}$. Denote by $N_{\alpha}$ the number of particles of the $\alpha$-species and put $\mathbf{N}=\left(N_{1}, \ldots, N_{S}\right)$. Obviously $\sum_{\alpha=1}^{S} N_{\alpha}=N=$ total particle number. Let $\left(z_{\alpha}, M_{\alpha}\right)$ be the charges and mass of species $\alpha$ and set $\mathbf{Z}=\left(z_{1}, \ldots, z_{S}\right)$ and $\mathbf{M}=\left(m_{1}, \ldots, m_{S}\right)$. Assume for simplicity that we have only two species of negative particles (namely electrons 
with "spin up" and electrons with "spin down"). Denote their common mass by $m$ while their common charge is set equal to -1 . To fix our notation, we choose $z_{1}=z_{2}=-1$ and $m_{1}=m_{2}=m$. The charge of the positive particles are assumed to be greater than or equal to 1 . We leave the masses of the positive particles unspecified but one should think of them as being much larger than $m$. This is the situation in the case of electrons and nuclei. Let $M$ be the total mass and let $Q$ be the total charge,

$$
M=\sum_{\alpha=1}^{S} N_{\alpha} m_{\alpha} \equiv \mathbf{N} \cdot \mathbf{M}, \quad Q=\sum_{\alpha=1}^{S} N_{\alpha} z_{\alpha} \equiv \mathbf{N} \cdot \mathbf{Z} .
$$

The quantum Coulomb Hamiltonian for $N$ particles can now be defined by

$$
H_{\mathrm{N}}=-\sum_{i=1}^{N} \frac{\Delta_{i}}{2 m_{i}}+\sum_{1 \leqq i<j \leqq N} \mathbf{e}_{i} \mathbf{e}_{j}\left|x_{i}-x_{j}\right|^{-1},
$$

where $\Delta_{i}$ denotes the Laplace operator in the variable $x_{i}$ and $\left(m_{i}, \mathbf{e}_{i}\right)=\left(m_{\alpha}, z_{\alpha}\right)$ if the $i^{\text {th }}$ particle belongs to the $\alpha$-species. The Hamiltonian $H_{\mathbf{N}}$ acts on a Hilbert space $\mathfrak{H}_{\mathbf{N}}$ of wave functions $\psi\left(x_{1}, \ldots, x_{N}\right) \in L^{2}\left(\mathbf{R}^{3 N}\right)$ which satisfies Fermi statistics for the two kinds of negative particles. The statistics of the positive particles are not relevant to our study and we shall make no assumption about their statistics. Fermi statistics means only that $\psi\left(x_{1}, \ldots, x_{n} \ldots\right)$ is antisymmetric when two electron's coordinates of the same species (i.e. same spin) are exchanged.

Let $\Lambda$ be a cube in $\mathbf{R}^{3}$. We shall also be interested in $H_{\mathbf{N}}$ acting on functions $\psi \in L^{2}\left(\Lambda^{3 N}\right)$ which satisfy Dirichlet boundary conditions on $\Lambda$. In that case we denote the restricted Hamiltonian by $H_{\Lambda, \mathrm{N}}$.

The Fock space mentioned in the beginning is defined as the direct sum of all the $\mathfrak{H}_{\mathbf{N}}$ 's. The Hamiltonian $H_{\mathbf{N}}$ can be naturally extended to the Fock space by requiring its restriction to $\mathfrak{H}_{N}$ be given by $H_{\mathbf{N}}$ in (1.2). We shall denote this Hamiltonian by $H$. Similarly, we can define $H_{\Lambda}$. In Fock space, the number of particles of the $\alpha^{\text {th }}$ species, $N_{\alpha}$, can be thought of as an operator (still denoted by $N_{\alpha}$ ) with eigenvalues $N_{\alpha}$. With this definition, $\mathfrak{H}_{N}$ is an eigenspace of the operator $N$. Similarly, we can define mass operators and charge operators etc. In this paper, we shall not be very careful to distinguish operators and numbers and shall use the same symbols for both.

Let us recall the basic definitions of the quantum grand canonical ensemble which is our main concern in this paper. Denote the chemical potential of the $\alpha$ species by $\mu_{\alpha}$ and put $\boldsymbol{\mu}=\left(\mu_{1}, \ldots, \mu_{S}\right)$. A fundamental quantity from which other thermodynamic quantities can be derived is the partition function. In the cube $\Lambda$ at inverse temperature $\beta$ it is defined by

$$
\Xi(\beta, \boldsymbol{\mu}, \Lambda)=\operatorname{Tr} \exp \left[\beta\left(\boldsymbol{\mu} \cdot \mathbf{N}-H_{\Lambda}\right)\right]=\sum_{\mathbf{N}} \operatorname{Tr} \exp \left[\beta\left(\boldsymbol{\mu} \cdot \mathbf{N}-H_{\Lambda, \mathbf{N}}\right)\right],
$$

where $\boldsymbol{\mu} \cdot \mathbf{N}=\sum_{\alpha=1}^{S} \mu_{\alpha} N_{\alpha}$. The corresponding pressure $p_{\Lambda}(\beta, \boldsymbol{\mu})$ is defined by

$$
p_{\Lambda}(\beta, \boldsymbol{\mu})=\beta^{-1} \log \Xi(\beta, \boldsymbol{\mu}, \Lambda) /|\Lambda| .
$$

A basic fact, proved by Lieb and Lebowitz [8] (see also [6]) is that the thermodynamic limit exists, i.e.

$$
p(\beta, \boldsymbol{\mu})=\lim _{\Lambda \rightarrow \infty} p_{\Lambda}(\beta, \boldsymbol{\mu})
$$


exists (if the limit $\Lambda \rightarrow \infty$ is taken in a proper sense) and the resulting function $p(\beta, \mu)$ depends on $\boldsymbol{\mu}$ only through the projection of $\boldsymbol{\mu}$ to the orthogonal complement of $\mathbf{Z}$, i.e., if we let $\boldsymbol{\mu}=\mu+\bar{\mu} \mathbf{Z}$ with $\bar{\mu}=(\boldsymbol{\mu} \cdot \mathbf{Z})(\mathbf{Z} \cdot \mathbf{Z})^{-1}$ (note that $\mu$ is a vector and $\bar{\mu}$ is a number) then, with a slight abuse of notation, we can write

$$
p(\beta, \boldsymbol{\mu})=p(\beta, \mu) .
$$

Another basic fact proved by Lieb and Lebowitz $[6,8]$ is that the thermodynamic quantities (such as the pressure) calculated [as in (1.5), for example] agree with those constructed in the canonical and in the microcanonical ensembles. An analogous theorem for correlation functions does not exist.

The relation (1.6) is indeed a statement about total charge neutrality. Another statement of charge neutrality is that one can restrict the summation in (1.3) to the neutral ensemble $Q=0$ but still obtain the same thermodynamic pressure. In short, there is no way to choose the chemical potentials so that the system fails to be neutral in the thermodynamic limit. See $[6,8]$ for a more detailed discussion. The advantage of (1.6) lies in the great technical simplifications it provides. Unlike the constraint $Q=0,(1.6)$ is easy to use as we shall see later. We should point out, however, that (1.5) and (1.6) are not obvious and their proofs are length.

Another thermodynamic quantity of interest is the density of the system. It is defined by

$$
\varrho(\beta, \boldsymbol{\mu})=\lim _{\Lambda \rightarrow \infty} \sum_{\alpha=1}^{S}\left[\partial_{p_{\Lambda}}(\beta, \boldsymbol{\mu}) / \partial \mu_{\alpha}\right] .
$$

It is known $[6,7]$ that

$$
\varrho(\beta, \boldsymbol{\mu})=\sum_{\alpha=1}^{S} \partial p(\beta, \boldsymbol{\mu}) / \partial \mu_{\alpha}
$$

for almost every $\boldsymbol{\mu}$, and $\varrho(\beta, \boldsymbol{\mu})$ depends on $\boldsymbol{\mu}$ also through $\mu$ alone.

Our main result in this paper, roughly speaking, is the proof that for low temperature $\beta^{-1}$ and low density $\varrho$ the thermodynamic pressure and density defined by (1.5) and (1.7) are given by a free gas of certain specific molecules (or atoms). The types of molecules are determined by the "ground states" of $H-\boldsymbol{\mu} \cdot \mathbf{N}$. Strictly speaking, $H-\boldsymbol{\mu} \cdot \mathbf{N}$ has no ground states because it is translation invariant. It is possible, however, to define the notion of "ground states" in terms of the "relative Hamiltonian" as follows. We write $H=H^{c}+H^{R}$, where $H_{\mathrm{N}}^{c}$ is given by

$$
H_{\mathbf{N}}^{c}=\frac{-1}{2 M} \Delta_{c}
$$

and $\Delta_{c}$ is the Laplacian in the center of mass co-ordinate $c$,

$$
c=\frac{1}{M} \sum_{i=1}^{N} m_{i} x_{i}
$$

Note that the relative Hamiltonian $H^{R}$ is only nontrivial on subspaces with at least 2 particles. Fix a chemical potential satisfying for some positive constant $\sigma$

$$
\begin{gathered}
H_{\mathbf{N}}-\mu \cdot \mathbf{N}>\sigma N, \\
\mu_{1}=\mu_{2}, \quad \mu \cdot \mathbf{Z}=0 .
\end{gathered}
$$


Define $\mathbf{e}_{0}(\mu)$ to be the infimum of the spectrum of $H^{R}-\mu \cdot \mathbf{N}$ with $\mathbf{N} \neq 0$ (i.e. the vacuum is not allowed). By assumption (1.11),

$$
\mathbf{e}_{0}(\mu)=\inf \operatorname{spec} H^{R}-\mu \cdot \mathbf{N}>0 \text {. }
$$

We claim that $\mathbf{e}_{0}(\mu)$ is an isolated eigenvalue of finite degeneracy if $\mu$ satisfies (1.12). To see this, by assumption (1.11) we have that the infimum of $H^{R}-\mu \cdot \mathbf{N}$ occurs only when all $N_{\alpha}$ are not too large. In other words, the cardinality of the set

$$
\Omega=\left\{\mathbf{N} \mid E_{\mathbf{N}} \equiv \inf \operatorname{spec} H_{\mathbf{N}}^{R}-\mu \cdot \mathbf{N}=\mathbf{e}_{0}(\mu)\right\}
$$

is finite. To establish our claim, it then remains to show that $E_{\mathbf{N}}$ is an isolated eigenvalue of $H_{\mathbf{N}}^{R}-\mu \cdot \mathbf{N}$ for all $\mathbf{N} \in \Omega$. But by the HVZ Theorem [3] the continuum spectrum of $H_{\mathbf{N}}^{R}-\mu \cdot \mathbf{N}$ starts from the infimum of the spectrum of all possible cluster decompositions (into at least two components) of $H_{\mathbf{N}}^{R}-\mu \cdot \mathbf{N}$. Our definition of $\mathbf{e}_{0}(\mu)$ then tells us this infimum is at least bigger than $2 \mathbf{e}_{0}(\mu)$. Hence $E_{\mathbf{N}}$ is not in the continuum spectrum and we can use general results $[3,9]$ to conclude that $E_{\mathbf{N}}$ is an isolated eigenvalue with finite degeneracy.

As a byproduct of the above discussion we have the following result: Let $\mu$ satisfy (1.12) and let $\mathbf{e}_{0}(\mu)$ be the ground state energy of $H^{R}-\mu \cdot \mathbf{N}$. Then there exists a constant $c_{1}$ depending on $H$ and $\mu$ such that if $\left|\mu^{\prime}-\mu\right|<c_{1}$ then $\mathbf{e}_{0}(\mu)$ and $\mathbf{e}_{0}\left(\mu^{\prime}\right)$ have the same eigenstates and

$$
\mathbf{e}_{0}(\mu)=\mathbf{e}_{0}\left(\mu^{\prime}\right)+\left(\mu^{\prime}-\mu\right) \cdot \mathbf{N} .
$$

The proof of (1.14) is almost the same as the arguments just given above. One first shows that the particle number can not be too big. Then choose $c_{1}$ to be (up to some constant) the gap between $\mathbf{e}_{0}(\mu)$ and the first excited state energy. We leave the details to the reader.

Let us denote the ground eigenstates by $\psi_{1}, \ldots, \psi_{g}$. Note that they depend on $\mu$ only in a global way, i.e., the $\psi_{i}$ do not change with respect to small perturbations of $\mu$. Denote their masses and numbers of particles by $\mathbf{M}^{(i)}$ and $\mathbf{N}^{(i)}$ with $i=1,2, \ldots, g$. Also, let

$$
M^{(i)}=\sum_{\alpha=1}^{S} M_{\alpha}^{(i)}, \quad N^{(i)}=\sum_{\alpha=1}^{S} N_{\alpha}^{(i)}, \quad Q^{(i)}=\mathbf{N}^{(i)} \cdot \mathbf{Z}
$$

be the total masses, particle numbers and charges. We may now state our basic assumption:

Assumption ( $A$ ). Fix a chemical potential $\mu$ satisfying (1.11) and (1.12). The ground states must either all be neutral (i.e. all $Q^{(i)}=0$ ) or else both signs of charge occur among $\psi_{1}, \ldots, \psi_{g}$. The latter case is referred to as the nonneutral case.

Generally, one expects in the nonneutral case that there are only two charges $Q_{+}>0$ and $Q_{-}<0$ such that $\left\{Q^{(i)} \mid i=1, \ldots, g\right\}=\left\{Q_{+}, Q_{-}\right\}$. We shall not need this assumption in the proof given below, but it is important for our proof that the set $\left\{Q^{(i)}\right\} \neq 0$ have both signs. Indeed, assumption $(A)$ is made for technical simplicity. Suppose the ground states do not satisfy our assumption, and suppose that the situation cannot be remedied by a small change in $\mu$. Then, for example, we will have only a charged ground state complex with a single charge, say $Q_{+}$. Clearly, we will not have an ideal gas of this complex. The neutrality requirement will force us to find another negatively charged complex to balance the Coulomb interaction. 
In this case, one should change $\mu$ to $\mu^{\prime}=\mu+\mu_{1} \mathbf{Z}$ and adjust $\mu_{1}$ such that our assumption holds. It is not very difficult to prove this is always possible and our proofs for Theorems 1.1 and 1.2 can then be carried out. The proofs are complicated, however, and will not be presented here.

Before stating the main theorems, let us introduce some notation and definitions. Since we shall use $H^{R}-\boldsymbol{\mu} \cdot \mathbf{N}$ to study $p(\beta, \mu)$, it is important to compare $H^{R}-\boldsymbol{\mu} \cdot \mathbf{N}$ and $H^{R}-\mu \cdot \mathbf{N}$. We shall assume that $\bar{\mu}$ is so small that the ground states of $H-\boldsymbol{\mu} \cdot \mathbf{N}$ and $H-\mu \cdot \mathbf{N}$ are the same. As we shall see later, we are only interested in the case when $\bar{\mu}$ is of order $1 / \beta$. Let $\mathbf{e}_{i}(\boldsymbol{\mu})$ be the energy of $\psi_{i}$ with respect to $H-\boldsymbol{\mu} \cdot \mathbf{N}$. Clearly,

$$
\mathbf{e}_{i}(\boldsymbol{\mu})=\mathbf{e}_{0}(\mu)+\bar{\mu} Q^{(i)}
$$

with $Q^{(i)}$ denoting the charge of $\psi^{(i)}$. Define two functions $G(\beta, \mu)$ and $R(\beta, \mu)$ by

$$
\begin{aligned}
G(\beta, \boldsymbol{\mu}) & =\frac{1}{\beta} \sum_{i=1}^{g}\left(\frac{M^{(i)}}{2 \pi \beta}\right)^{3 / 2} \exp \left[-\beta \mathbf{e}_{i}(\boldsymbol{\mu})\right], \\
R(\beta, \boldsymbol{\mu}) & =\sum_{i=1}^{g} N^{(i)}\left(\frac{M^{(i)}}{2 \pi \beta}\right)^{3 / 2} \exp \left[-\beta \mathbf{e}_{i}(\boldsymbol{\mu})\right] .
\end{aligned}
$$

The function $G$ can be thought of as (approximately) the pressure of a free gas of particles with mass $M^{(i)}$ and "internal energy" $\mathbf{e}_{i}(\mu)$ (cf. Sect. 2). Similarly, $R$ is the number density. The function $G$ is convex in $\bar{\mu}$ (strictly convex if some $Q^{(i)} \neq 0$ ) and has a unique minimizer with respect to $\bar{\mu}$ [denoted by $\omega(\mu)$ ] if $Q^{(i)} Q^{(j)}<0$ for some $i \neq j$. The derivative of $G$ with respect to $\bar{\mu}$ can be interpreted as the charge density, and we shall denote it by $F(\beta, \mu)$.

We can define a function $g(\beta, \mu)$ by

$$
g(\beta, \mu)=\inf _{\tilde{\mu}} G(\beta, \boldsymbol{\mu})=G(\beta, \mu+\omega(\mu) \mathbf{Z}) .
$$

A minimizer for (1.18) always exists and is unique in the nonneutral case $\left(Q^{(i)} Q^{(j)}<0\right)$. We extend the definition to the neutral case by defining $\omega(\mu)=0$, hence

$$
\omega(\mu)=\left\{\begin{array}{l}
0 \quad \text { in the neutral case } \\
\text { The unique solution of } F(\beta, \mu+\omega \mathbf{Z})=0, \text { non neutral case. }
\end{array}\right.
$$

Since $G$ is just a linear combination of exponential functions, it is not difficult to check that for $\beta$ large

$$
\omega(\mu)=O(1 / \beta) .
$$

We also need a function $r(\beta, \mu)$ defined by

$$
r(\beta, \mu)=R(\beta, \mu), \quad \bar{\mu}=\omega(\mu) .
$$

We may now state our main results as follows.

Theorem 1.1. Suppose assumption $(A)$ holds. Then there exist $\varepsilon>0, \beta_{0}>0$ depending on $\mu$ and $H$ such that if $\beta \geqq \beta_{0}$, the thermodynamic pressure is given by the equation

$$
\beta p(\beta, \mu)=g(\beta, \mu)\left[1+O\left(e^{-\varepsilon \beta}\right)\right] .
$$


Theorem 1.2. With the same assumptions as in Theorem 1.1 the thermodynamic density satisfies the following equation for almost every $\beta$ and $\mu$,

$$
\varrho(\beta, \mu)=r(\beta, \mu)\left[1+O\left(e^{-\varepsilon \beta}\right)\right] .
$$

To gain perspective on the content of our theorems let us consider the simplest case of the electron-proton system. It is convenient in this case to write $\boldsymbol{\mu}=\mu\left(\frac{1}{2}, \frac{1}{2}, 1\right)$. Note that $\mu$ is a scalar. Let us define

$$
E_{N}=\inf \left\{\inf \operatorname{spec}\left(H_{N+, N_{-}}^{R}\right): N_{+}+N_{-}=N\right\}
$$

and denote the convex hull of $\left\{\left(N, E_{N}\right) \mid N \geqq 1\right\}$ by $K$. It is presumably the case (as Fefferman conjectures) that $(2, E(2))$ is in $K$. If so, what $\mu$ will select the hydrogen atom? We must have $E(1)-\mu>E(2)-2 \mu>0$ and $0<E(2)-2 \mu<E(N)-N \mu$ for all $N \geqq 3$. The first condition gives $\mu>E(2)=-\frac{1}{4}$. The second condition (if we make a rough, and so far unprovable guess) is

$$
\mu<E(3)-E(2) \approx \frac{3}{2}\left(-\frac{1}{4}\right)-\left(-\frac{1}{4}\right)=-\frac{1}{8} .
$$

On the other hand, if $\mu<E(2)$ then the first inequality reverses and we pick up the extremal point $(1, E(1))$. Clearly, $(1, E(1))$ corresponds to complete ionization. In both cases, it is not difficult to check that Theorem 1.1 and Theorem 1.2 yield the ideal gas equations $\beta p=\frac{1}{2} \varrho$ (the hydrogen atom case) and $\beta p=\varrho$ (the complete ionization case) - as expected.

Our method of proof for Theorems 1.1 and 1.2 consists in obtaining upper and lower bounds on partition functions. The proof of the lower bound follows the ideas of Lieb and Lebowitz [8] by applying the Peierls-Bogoliubov inequality. For the proof of the upper bound we use the localization method of [2]. In theorems 1.1 and 1.2 the correction terms from perfect gas behavior are $O\left(e^{-\varepsilon \beta}\right)$ whereas in the work of Fefferman correction terms are $O(1 / \beta)$. The reason that we are able to obtain sharper estimates than [5] is that we use the localization method to bound the Coulomb Hamiltonian from below by a localized Yukawa Hamiltonian. Fefferman bounds the Coulomb Hamiltonian from below by a localized Coulomb-like Hamiltonian.

To illustrate why the use of a Yukawa Hamiltonian has advantages we consider a simple inequality. Let $\Xi_{v}$ denote the partition function which is defined exactly as in (1.3) but with the Coulomb interaction $1 /|x|$ replaced by the Yukawa interaction $Y_{v}(x)=e^{-v|x|} /|x|$ with $v>0$. If we observe now that the function $\phi(x)$ $=\left(1-e^{-v|x|}\right) /|x|$ is positive definite (in the sense that its Fourier transform is positive) and satisfies $\phi(0)=v$ it follows that

$$
\sum_{1 \leqq i<j \leqq N} \mathbf{e}_{i} \mathbf{e}_{j} \phi\left(x_{i}-x_{j}\right) \geqq-\frac{1}{2} v \sum_{i=1}^{N} \mathbf{e}_{i}^{2},
$$

for charges $\mathbf{e}_{i}$ at positions $x_{i} \in \mathbf{R}^{3}, 1 \leqq i \leqq N$. Thus if $\Xi$ denotes the Coulomb partition function we have the inequality

$$
\Xi(\beta, \boldsymbol{\mu}, \Lambda) \leqq \Xi_{v}\left(\beta, \boldsymbol{\mu}+\frac{1}{2} \mathbf{v}, \Lambda\right),
$$

where $\mathbf{v}$ denotes the vector, $\mathbf{v}=v\left(z_{1}^{2}, \ldots, z_{S}^{2}\right)$. Now the system with Yukawa interaction has short range interactions and it has been shown by Brydges and Federbush [1] that the partition function $\Xi_{v}$ can be expanded in a cluster 
expansion provided $\mu$ is sufficiently negative. This cluster expansion will give terms like those which occur in Theorem 1.1 plus other terms which are $O\left(e^{-\varepsilon \beta}\right)$. If we choose $v=O\left(e^{-\varepsilon \beta}\right)$ then the inequality (1.24) also generates correction terms of $O\left(e^{-\varepsilon \beta}\right)$. Therefore if we put the inequality (1.24) together with the results of Brydges and Federbush [1] we obtain an upper bound on the pressure of the form given in Theorem 1.1 provided $\mu$ is sufficiently negative.

Since we intend to bound the Coulomb partition function by a localized Yukawa partition function we need to prove that assumption $(A)$ is stable under perturbation of the Coulomb-interaction to Yukawa interaction with small $v$. Let $H_{\mathrm{N}}^{v}$ denote the Hamiltonian which is the same as (1.2) except that the Coulomb interaction is replaced by the Yukawa $Y_{v}$, and let $H^{v}$ be the corresponding Fock space Hamiltonian.

In the following theorem, let $M=\max \left\{m_{\alpha} \mid \alpha=1, \ldots, S\right\}$.

Theorem 1.3. Suppose there exist $\mu$ and $\sigma>0$ such that $H-\mu \cdot \mathbf{N}>\sigma N$ for all particle numbers $\mathbf{N}$. Then there is universal constant $c_{1}$ such that if $0<v / M<\frac{1}{2}$, then

$$
H^{\nu}-\mu \cdot \mathbf{N}>\left[\sigma-c_{1} M|\log (v / M)|^{-1 / 6}\right] N .
$$

Corollary. Fix $\mu$ satisfying assumption $(A)$. Then for $v$ small enough assumption $(A)$ holds with the same $\mu$ if we replace $H$ by $H^{v}$ and $\sigma$ by $\sigma-c_{1} M|\log (v / M)|^{-1 / 6}$ in the following sense: 1.

$$
H^{v}-\mu \cdot \mathbf{N}>\left(\sigma-c_{1} M|\log (v / M)|^{-1 / 6}\right) N .
$$

2. The lowest $g$ values (counting multiplicity) of the spectrum of $H^{v, R}-\mu \cdot \mathbf{N}$ are discrete (denote them by $\mathbf{e}_{1}^{\mathrm{v}}(\mu) \leqq \mathbf{e}_{2}^{\mathrm{v}}(\mu) \leqq \ldots \leqq \mathbf{e}_{g}^{\mathrm{v}}(\mu)$ ) and can be bounded for some constant $c_{2}$ by

$$
\left|\mathbf{e}_{j}^{v}(\mu)-\mathbf{e}_{0}(\mu)\right| \leqq c_{2} v .
$$

3. Let $\tau$ be the gap between $\mathbf{e}_{0}(\mu)$ and the first excited energy of $H^{R}-\mu \cdot \mathbf{N}$. Then there is a gap $\tau^{\prime}>\tau-c_{2} v$ between $\mathbf{e}_{g}^{v}(\mu)$ and the $g+1$ eigenvalue (or continuum spectrum) of $H^{v, R}-\mu \cdot \mathbf{N}$.

The proof of this corollary is simple. Let $E_{\mathrm{N}}(v)$ denote the infimum of the spectrum of $H_{\mathrm{N}}^{v, R}$. Then since $\left|r^{-1}-r^{-1} e^{-v r}\right| \leqq v$ for all $r$, we immediately have the operator bound

$$
\left|H_{\mathrm{N}}^{v, R}-H_{\mathrm{N}}^{R}\right| \leqq \frac{1}{2} v N(N-1) .
$$

Suppose $v$ is so small that

$$
c_{1} M|\log (v / M)|^{-1 / 6}<\sigma / 2 .
$$

Then for $N>N_{0}=2\left(\mathbf{e}_{0}(\mu)+2 \tau\right) / \sigma$ we have from Theorem 1.3 that $E_{\mathbf{N}}(v)-\mu \cdot \mathbf{N}$ $\geqq \frac{\sigma}{2} N \geqq \mathbf{e}_{0}(\mu)+2 \tau$. The statements (2) and (3) then follow from the above remark and the operator bound (1.28) if we choose $v$ satisfying (1.29) and

$$
c_{2}=\frac{1}{2} N_{0}^{2} \text {. }
$$

Theorem 1.3 will be proved in Sect. 5. Again, we use the localization method in [2]. While the bound (1.25) is sufficient for our purpose to prove Theorems 1.1 and 1.2 , it is clearly not optimal in the dependence on $v$. In view of (1.28) we expect a linear dependence on $v$. We hope the interested reader will prove this. 


\section{Bounds on the Partition Function in a Fixed Box}

We wish to find upper and lower bounds on the partition function in a box of fixed size. First we need a lemma concerning the eigenvalues of the relative Hamiltonian $H^{R}$. Let $H^{R, a}$ denote the relative Hamiltonian restricted to the ball of radius $a$. Thus $H_{\mathbf{N}}^{R, a}$ acts on wave functions $\psi\left(x_{1}, \ldots, x_{N}\right)$ which are supported in the set

$$
\sum_{i=1}^{N} m_{i} x_{i}=0, \quad\left|x_{i}\right| \leqq a, \quad i=1, \ldots, N
$$

and satisfy Dirichlet boundary conditions on $\left|x_{i}\right|=a, i=1, \ldots, N$.

Lemma 2.1. Suppose $\mathbf{e}_{0}(\boldsymbol{\mu})$ is the ground state energy of $H^{\boldsymbol{R}}-\boldsymbol{\mu} \cdot \mathbf{N}$ with $g$ degeneracy. Let $E_{1}(a), \ldots, E_{g}(a)$, be the first $g$ eigenvalues of $H^{R, a}-\boldsymbol{\mu} \cdot \mathbf{N}$. Then there exists $a$ constant $c_{3}$ such that for $a>c_{3}$ we have

$$
\mathbf{e}_{0}(\boldsymbol{\mu}) \leqq E_{i}(a) \leqq \mathbf{e}_{0}(\boldsymbol{\mu})+c_{4} a^{-2}, \quad i=1, \ldots, g,
$$

for some constant $c_{4}$ independent of $a$.

Proof. The lower bound of (2.2) is obvious since imposing Dirichlet boundary conditions can only increase energy. For the upper bound, it is convenient to prove instead

$$
\sum_{i=1}^{g} E_{i}(a) \leqq g \mathbf{e}_{0}(\boldsymbol{\mu})+c_{4} a^{-2}
$$

for some constant $c_{4}$. Recall that $\psi_{i}, i=1, \ldots, g$, are the ground states of $H^{R}-\boldsymbol{\mu} \cdot \mathbf{N}$. Define $g$ functions in the domain of $H^{R, a}-\boldsymbol{\mu} \cdot \mathbf{N}$ by

$$
\psi_{j}^{a}=\psi_{j}\left(x_{1}, \ldots, x_{N}\right) \prod_{i=1}^{N} \chi\left(x_{i}\right),
$$

where $\chi$ is a smooth cutoff function satisfying

$$
\chi(x)= \begin{cases}1 & |x| \leqq a / 2 \\ 0 & |x| \geqq a\end{cases}
$$

Denote their overlaps by

$$
L_{i j}=\left\langle\psi_{i}^{a}, \psi_{j}^{a}\right\rangle .
$$

Clearly, there is a constant $c_{3}>0$ such that for $a>c_{3}$ one has that $A \equiv L^{-1 / 2}$ exists and is bounded in the norm $\|A\|=\sup \left|A_{i j}\right|$. Denote by $\varrho(x, y)$ the matrix defined by

$$
\varrho(x, y)=\sum_{i, j, k} \bar{A}_{i k} \overline{\psi_{k}^{a}(y)} \psi_{j}^{a}(x) A_{i j} .
$$

It is easy to check that $\varrho$ is a $g$-dimensional projection. Now by the variational principle we have

$$
\begin{aligned}
\sum_{i=1}^{g} E_{i}(a) \leqq & \operatorname{Tr} \varrho\left(H^{R, a}-\boldsymbol{\mu} \cdot \mathbf{N}\right) \leqq \sum_{i, j, k}\left\{\overline{A_{i j} \overline{A_{i k}}} \int\left[\prod_{i=1}^{N} \chi\left(x_{i}\right)\right]^{2}\right. \\
& \left.\times \psi_{j}\left(H^{R, a}-\boldsymbol{\mu} \cdot \mathbf{N}\right) \psi_{k} d x+A_{i j} A_{i k} \sup |\nabla \chi|^{2}\right\}
\end{aligned}
$$


where we have used the identity

$$
\int \chi f(-\Delta) \chi f d x=\int \chi^{2} f(-\Delta) f d x+\int|\nabla \chi|^{2} f^{2} d x .
$$

Now by using the fact that $\psi_{k}$ is an eigenstate, we find that the first term in the right side of (2.8) is just $g \mathbf{e}_{0}(\mu)$. The second term is easily seen to give the corrections $c_{4} a^{-2}$.

Remark. The constant $c_{3}$ in Lemma 2.1 depends on the rate of fall off of the eigenfunctions $\psi_{i}$. In the appendix we prove a similar result where $c_{3}$ depends only on the eigenvalues of $H$ and $\mu$.

Let $U_{\varepsilon}$ be a cube in $R^{3}$ with side of length $e^{5 \varepsilon \beta}$ where $\varepsilon>0$ and $\beta$ is large. We wish to find a lower bound for the grand partition function on $U_{\varepsilon}$. To do this we define an ensemble on $U_{\varepsilon}$ which consists of a subset of the configurations of the grand canonical ensemble on $U_{\varepsilon}$. We shall denote this new ensemble by $G$. To define it we take $a=e^{4 \varepsilon \beta}$ in Lemma 2.1 and let $\phi_{i}^{a}, i=1, \ldots, g$, be the eigenfunctions of $H^{R, a}-\boldsymbol{\mu} \cdot \mathbf{N}$ corresponding to the eigenvalues $E_{i}(a), i=1, \ldots, g$ respectively. By Lemma $2.1 E_{i}(a)$ can be bounded by

$$
E_{i}(a)=\mathbf{e}_{0}(\boldsymbol{\mu})+O\left(e^{-\varepsilon \beta}\right) .
$$

Suppose that $\bar{\mu}|\mathbf{Z}|=|\mu-\boldsymbol{\mu}|=O(1 / \beta)$ and $\beta$ is large enough. Then, as remarked in Sect. 1, [cf. (1.15)] the ground states $\left\{\varphi_{i}\right\}$ for $H-\mu \cdot \mathbf{N}$ are also ground states for $H-\boldsymbol{\mu} \cdot \mathbf{N}$. Furthermore, the masses, charges and particle numbers for $\left\{\varphi_{i}^{a}\right\}$ are the same as for $\left\{\varphi_{i}\right\}$. Thus we shall use $M^{(i)}, N^{(i)}$, and $Q^{(i)}$ for both $\varphi_{i}$ and $\varphi_{i}^{(a)}$. Now, for $k$ varying in the positive octant of $\mathbf{Z}^{3}$, let $f_{k}$ be the eigenfunctions of the Laplace operator with Dirichlet boundary conditions on the cube concentric with $U_{\varepsilon}$ but with side of length $l=e^{5 \varepsilon \beta}-3 e^{4 \varepsilon \beta}$. Thus

$$
-\Delta f_{k}=\lambda_{k}^{2} f_{k}
$$

where

$$
\lambda_{k}=\pi|k| e^{-5 \varepsilon \beta}\left[1-3 e^{-\varepsilon \beta}\right]^{-1} .
$$

We then define normalized wave functions $\psi_{k, i}$ by

$$
\psi_{k, i}\left(x_{1}, \ldots, x_{N_{i}}\right)=f_{k}(c) \varphi_{i}^{a}\left(x_{1}-c, \ldots, x_{N_{i}}-c\right),
$$

and $c$ is the center of mass of the $x_{j}, 1 \leqq j \leqq N_{i}$ which is defined in (1.11). Evidently the $\psi_{k, i}$ form an orthonormal set supported in $\left(U_{\varepsilon}\right)^{N_{i}}$ and

$$
(H-\boldsymbol{\mu} \cdot \mathbf{N}) \psi_{k, i}=\left[\frac{\lambda_{k}^{2}}{2 M_{i}}+E_{i}(a)\right] \psi_{k, i} .
$$

The partition function for the ensemble $G$ is given by

$$
\Xi_{G}\left(\beta, \boldsymbol{\mu}, U_{\varepsilon}\right)=1+\sum_{k, i} \exp \left[\beta\left\langle\psi_{k i},\left(\bar{\mu} Q^{(i)}+\mu \cdot \mathbf{N}-H\right) \psi_{k i}\right\rangle\right] .
$$

Clearly, one has the bound (by the Peierls inequality [11, Proposition 2.5.4])

$$
\Xi\left(\beta, \boldsymbol{\mu}, U_{\varepsilon}\right) \geqq \Xi_{G}\left(\beta, \boldsymbol{\mu}, U_{\varepsilon}\right) .
$$

The partition function $\Xi_{G}\left(\beta, \mu, U_{\varepsilon}\right)$ is easy to estimate. By using the bound

$$
l^{-3} \sum_{k} \exp \left[-\alpha \lambda_{k}^{2}\right]=(2 \pi \alpha)^{-3 / 2}+O\left(l^{-1}\right)
$$


we have

$$
\begin{aligned}
& \left|U_{\varepsilon}\right|^{-1} \log \Xi_{G}\left(\beta, \mu, U_{\varepsilon}\right) \geqq \sum_{i=1}^{g}\left[\left(\frac{M^{(i)}}{2 \pi \beta}\right)^{3 / 2}+O\left(e^{-\varepsilon \beta}\right)\right] \\
& \quad \times \exp \left\{-\beta\left[\mathbf{e}_{i}(\mu)+\bar{\mu} Q^{(i)}+O\left(e^{-\varepsilon \beta}\right)\right]\right\} .
\end{aligned}
$$

Putting (2.16) and (2.18) together, we have proved the following:

Lemma 2.2. Suppose $\mu$ satisfies assumption $A$ and $\bar{\mu}=O(1 / \beta)$. Then there exist $\beta_{0}>0$, $\varepsilon_{1}$ and $\varepsilon_{2}$ such that if $\beta>\beta_{0}$ and if $\varepsilon_{1}<\varepsilon<\varepsilon_{2}$, then

$$
p_{U_{\varepsilon}}(\beta, \boldsymbol{\mu}) \geqq G(\beta, \boldsymbol{\mu})\left[1+O\left(e^{-\varepsilon \beta}\right)\right] .
$$

Next we obtain an upper bound for the pressure. The proof of this lemma uses an idea from Fefferman [5] and Lieb-Lebowitz [8, Theorem 2.2].

Lemma 2.3. Let $\beta, \mu$, and $\varepsilon$ satisfy the conditions of Lemma 2.2 (with possibly different $\beta_{0}, \varepsilon_{1}$, and $\varepsilon_{2}$ ). Then the pressure can be bounded above by

$$
p_{U_{\varepsilon}}(\beta, \boldsymbol{\mu}) \leqq G(\beta, \boldsymbol{\mu})\left[1+O\left(e^{-\varepsilon \beta}\right)\right] .
$$

Proof. Let $K_{\mathbf{N}}$ be the kinetic energy defined by

$$
K_{\mathrm{N}}=-\sum_{i=1}^{N}\left(\Delta_{i} / 2 m_{i}\right)
$$

Write $H_{\mathbf{N}}=(1-\delta) K_{\mathbf{N}}+V_{c}+\delta K_{\mathbf{N}}\left(V_{c}\right.$ is the Coulomb energy part of $\left.H_{\mathbf{N}}\right)$. By scaling we have

$$
(1-\delta) K_{\mathbf{N}}+V_{c} \geqq(1-\delta)^{-1} E_{\mathbf{N}},
$$

where $E_{\mathbf{N}}=\inf \operatorname{spec} H_{\mathbf{N}}$. Hence from (1.11) $H_{\mathbf{N}}-\boldsymbol{\mu} \cdot \mathbf{N}$ can be bounded below by

$$
H_{\mathbf{N}}-\boldsymbol{\mu} \cdot \mathbf{N} \geqq \delta K_{\mathbf{N}}+\left[\sigma N-\delta(1-\delta)^{-1} \mu \cdot \mathbf{N}-\bar{\mu} Q\right],
$$

where $Q$ is defined by $Q=\mathbf{Z} \cdot \mathbf{N}$. Choose $\delta$ satisfying $\delta(1-\delta)^{-1}\left(\max \mu_{i}\right) \leqq \sigma / 4$. Recall that $\bar{\mu}=O\left(\beta^{-1}\right)$ whence for $\beta$ large enough

$$
H_{\mathbf{N}}-\boldsymbol{\mu} \cdot \mathbf{N} \geqq \delta K_{\mathbf{N}}+\sigma N / 2 .
$$

Equation (2.24) also holds if we replace $H_{\mathrm{N}}$ and $K_{\mathrm{N}}$ by $H_{\mathrm{N}}^{a}$ and $K_{\mathrm{N}}^{a}$ respectively. To check this statement, one only has to note that (2.22) holds for $K_{\mathrm{N}}^{a}$ replacing $K_{\mathrm{N}}$. Thus one has

$$
\operatorname{Tr} \exp \left[\beta\left(\mu \cdot \mathbf{N}-H_{U_{\varepsilon}, \mathbf{N}}\right)\right] \leqq \prod_{i=1}^{N}\left(\frac{m_{i}}{2 \pi \beta \delta}\right)^{3 / 2}\left|U_{\varepsilon}\right|^{N} \exp [-\beta \sigma N / 4]
$$

for $\beta$ sufficiently large. Since $\left|U_{\varepsilon}\right| \sim e^{15 \varepsilon \beta}$, by choosing $\varepsilon<\sigma / 120$ the last two factors in (2.25) can be bounded by $\exp (-\beta \sigma N / 120)$. The first factor in $(2.25)$ is obviously smaller than $\exp (\beta \sigma N / 240)$ for large $\beta$ and thus

$$
\operatorname{Tr} \exp \left[\beta\left(\boldsymbol{\mu} \cdot \mathbf{N}-H_{U_{\varepsilon}, \mathbf{N}}\right)\right] \leqq \exp [-\beta \sigma N / 240] .
$$

But $\beta G(\beta, \mu) \geqq O\left(e^{-\alpha \beta}\right)$ for some fixed constant $\alpha$. Hence if we choose $N_{0}$ and $\varepsilon$ such that

$$
\sigma N_{0} / 240 \geqq \alpha+2 \varepsilon,
$$


then

$$
\left|U_{\varepsilon}\right|^{-1} \sum_{N \leqq N_{0}} \operatorname{Tr} \exp \left[\beta\left(\boldsymbol{\mu} \cdot \mathbf{N}-H_{U_{\varepsilon}, \mathbf{N}}\right)\right] \leqq G(\beta, \boldsymbol{\mu}) O\left(e^{-\varepsilon \beta}\right) .
$$

Next we consider the case $N<N_{0}$. Define a subspace $Y$ by

$$
Y=\bigoplus_{\mathbf{N} \in A}\left\{\mathfrak{H}_{U_{\varepsilon}, \mathbf{N}}\right\}, \quad A=\left\{\mathbf{N} \mid N<N_{0}, H_{\mathbf{N}}-\boldsymbol{\mu} \cdot \mathbf{N}>\mathbf{e}_{0}(\boldsymbol{\mu})+c\right\}
$$

where $c$ is the gap between the first excited energy $\mathbf{e}_{1}(\boldsymbol{\mu})$ and the ground state energy $\mathbf{e}_{0}(\boldsymbol{\mu})$ of $H-\boldsymbol{\mu} \cdot \mathbf{N}$. Here $\mathfrak{H}_{U_{\varepsilon}, \mathbf{N}}$ is the subspace of $\mathbf{N}$ particle wave functions with $x_{i} \in U_{\varepsilon}$ for all $i$. Again we split the kinetic energy into two pieces and use the simple fact $K_{\mathrm{N}}^{a} \geqq K_{\mathrm{N}}$ and (2.22) to yield

$$
H_{\mathbf{N}}^{a}-\boldsymbol{\mu} \cdot \mathbf{N} \geqq \delta K_{\mathbf{N}}^{a}+c-\delta(1-\delta)^{-1} \boldsymbol{\mu} \cdot \mathbf{N} .
$$

By choosing $\delta$ with $\delta(1-\delta)^{-1}\left(\max \mu_{i}\right) N_{0}<c / 2$ we conclude

$$
\operatorname{Tr}_{Y} \exp \left[\beta\left(\boldsymbol{\mu} \cdot \mathbf{N}-H_{U_{\varepsilon}, \mathbf{N}}\right)\right] \leqq \sum_{N<N_{0}} \prod_{i=1}^{N}\left(\frac{m_{i}}{2 \pi \beta \delta}\right)^{3 / 2}\left|U_{\varepsilon}\right|^{N} e^{-\beta c / 2} e^{-\beta \mathbf{e}_{0}(\boldsymbol{\mu})}
$$

Since $c>0$, the left side of (2.31) is again small in the sense of (2.28) if $\beta$ is sufficiently large. It remains to bound the contribution from the subspace $Y^{\prime}$ defined by

$$
Y^{\prime}=\bigoplus_{\mathbf{N} \in \boldsymbol{B}}\left\{\mathfrak{S}_{U_{\varepsilon}, \mathbf{N}}\right\}, \quad B=\left\{\mathbf{N} \mid N<N_{0}, \mathbf{N} \notin A\right\} .
$$

Let $X$ be the subspace defined by

with

$$
X \equiv L^{2}\left(U_{\varepsilon}\right) \otimes X_{R}
$$

$$
\begin{gathered}
X_{R} \equiv\left\{\psi\left(x_{1}, \ldots, x_{N}\right) \mid \psi\right. \text { are wave functions satisfying the statistics requirements } \\
\text { and } \left.x_{i} \in 2 U_{\varepsilon} \text { and } \sum_{i=1}^{N} m_{i} x_{i}=0 ; \mathbf{N} \in B\right\}
\end{gathered}
$$

Clearly, $Y^{\prime} \subset X$ with the natural injection from $Y^{\prime}$ to $X$. It follows that

$$
\operatorname{Tr}_{Y^{\prime}} \exp \left\{\beta\left(\boldsymbol{\mu} \cdot \mathbf{N}-H_{U_{\varepsilon}, \mathbf{N}}\right)\right\} \leqq \operatorname{Tr}_{X} \exp \left\{\beta\left(\boldsymbol{\mu} \cdot \mathbf{N}-H_{U_{\varepsilon}, N}\right)\right\} .
$$

Now let $X_{R, g}$ be the subspace of the first $g$ eigenstates of $H^{R}-\boldsymbol{\mu} \cdot \mathbf{N}$ and denote its orthogonal complement in $X_{R}$ by $X_{R, g}^{\perp}$. Again the contribution from $L^{2}\left(U_{\varepsilon}\right) \otimes X_{R, g}^{\perp}$ is small since the Hamiltonian $H^{R}-\boldsymbol{\mu} \cdot \mathbf{N}$ restricted to $X_{R, g}^{\perp}$ is larger than $\mathbf{e}_{0}(\boldsymbol{\mu})+c$. Finally, the contribution from $L^{2}\left(U_{\varepsilon}\right) \otimes X_{R, g}$ is easy to compute and can be bounded by

$$
\operatorname{Tr}_{L^{2}\left(U_{\varepsilon}\right) \otimes X_{R, g}} \leqq \beta\left|U_{\varepsilon}\right| G(\beta, \mu)\left(1+O\left(e^{-\varepsilon \beta}\right)\right) .
$$

We conclude Lemma 2.3 by putting all these bounds together.

Remark. It is not difficult to check that Lemma 2.3 also holds if we replace the Coulomb potential by a Yukawa potential with $v=O\left(e^{-\varepsilon \beta}\right)$. One only has to relate the Yukawa Hamiltonian to the Coulomb Hamiltonian by Theorem 1.3. 


\section{The Localization Method}

We wish to use the localization method developed in [2] to obtain an upper bound on the partition function. The main result in this section is the following Lemma 3.1. It was stated slightly differently in [2]. We shall give another proof since we emphasize a slightly different aspect here.

First we need to define a function $g(t)$ which is supported in an interval slightly larger than the unit interval $|t| \leqq \frac{1}{2}$ and such that

$$
\sum_{j \in Z} g^{2}(t+j)=1, \quad \text { all } t \in \mathbf{R} .
$$

To accomplish this let $f(s),-\infty<s<\infty$, be a $C^{\infty}$ function such that

$$
f(s)=\frac{1}{\sqrt{2}} \text { if } \quad s \leqq 0, \quad f(s)=0 \quad \text { if } \quad s \geqq 1 .
$$

For any given $\eta, 0<\eta<\frac{1}{2}$ we define $g(t)$ by

$$
\begin{gathered}
g(t)=\left[1-f^{2}\left(\frac{\frac{1}{2}-t}{\eta}\right)\right]^{1 / 2}, \quad 0 \leqq t \leqq \frac{1}{2}, \\
g(t)=f\left(\frac{t-\frac{1}{2}}{\eta}\right), \quad t \geqq \frac{1}{2}, \\
g(t)=g(-t), \quad t \in \mathbf{R} .
\end{gathered}
$$

It is clear that $g(t)$ is a $C^{\infty}$ function supported in the interval $|t| \leqq \frac{1}{2}+\eta$, and that (3.1) holds.

The function $g(t)$ can be used to generate a translation invariant partition of unity for $\mathbf{R}^{3}$. If we let $\chi(x)=g\left(x^{1}\right) g\left(x^{2}\right) g\left(x^{3}\right)$ with $x=\left(x^{1}, x^{2}, x^{3}\right)$ and put $\chi_{\lambda}(x)$ $=\chi(x+\lambda)$, where $\lambda \in \mathbf{Z}^{3}$, then it follows from (3.1) that

$$
\sum_{\lambda \in \mathbf{Z}^{3}} \chi_{\lambda}^{2}(x)=1, \quad \forall x \in \mathbf{R}^{3} .
$$

Let $\Gamma$ be the unit cube in $\mathbf{R}^{3}$ with center at the origin. A function $h$ which is of central importance to us is defined by

$$
\begin{aligned}
h(x, y) & =\int_{\Gamma} d u \sum_{\lambda \in \mathbf{Z}^{3}} \chi_{\lambda}^{2}(x+u) \chi_{\lambda}^{2}(y+u) \\
& =\int_{\mathbf{R}^{3}} d u \chi^{2}(x+u) \chi^{2}(y+u) .
\end{aligned}
$$

It is evident from (3.5) that $h(x, y)$ depends only on the difference $z=x-y$ and

$$
h(z)=h(x-y)=\chi^{2} * \chi^{2}(z) \text {. }
$$

It is obvious then that

$$
h(0)=\left[\int_{-\infty}^{\infty} g^{4}(t) d t\right]^{3}=1+O(\eta) .
$$

Furthermore $h(z)$ and its derivatives satisfy the inequality

$$
|h(z)| \leqq C, \quad\left|\partial^{\alpha} h(z)\right| \leqq \frac{C}{\eta^{|\alpha|-1}}, \quad|\alpha| \geqq 1,
$$

for some universal constant $C$. 
Now for $\lambda \in \mathbf{Z}^{3}$ and $l>0$ let $\mathfrak{S}_{l, \lambda}$ denote the Fock space for the cube with center $l \lambda$ and side of length $l(1+2 \eta)$. We introduce a Hamiltonian $H_{l, \lambda, \eta}^{v}$ on $\mathfrak{H}_{l, \lambda, \eta}$ which differs from the Coulomb Hamiltonian (1.1) by replacing the Coulomb interaction with a Yukawa and by including a scale factor depending on $\eta$. On an eigenspace of $\mathbf{N}$ it is given by

$$
H_{l, \lambda, \eta, \mathbf{N}}^{v}=-\sum_{i=1}^{N} \frac{1}{2 m_{i}} \Delta_{i}+h(0)^{-1} \sum_{1 \leqq i<j \leqq N} \mathbf{e}_{i} \mathbf{e}_{j} Y_{v}\left(x_{i}-x_{j}\right) .
$$

We assume this Hamiltonian acts on functions in $\mathfrak{H}_{l, \lambda, \eta}$ which satisfy Dirichlet boundary conditions. For any open subset $\Lambda$ in $\mathbf{R}^{3}$ we define a subset $Z^{3}(\Lambda, l, \eta)$ of $Z^{3}$ by

$$
\begin{aligned}
Z^{3}(\Lambda, l, \eta)= & \left\{\lambda \in \mathbf{Z}^{3}: \text { there exists } u \in \Gamma \text { such that the cube with center }(\lambda+u) l\right. \\
& \text { and side of length } l(1+2 \eta) \text { intersects } \Lambda\} .
\end{aligned}
$$

Then we define the Hilbert space $\mathfrak{H}_{l, \Lambda, \eta}$ by

$$
\mathfrak{H}_{l, \Lambda, \eta}=\bigotimes_{\lambda \in Z^{3}(\Lambda, l, \eta)} \mathfrak{H}_{l, \lambda, \eta}
$$

and the corresponding Hamiltonian $H_{l, \Lambda, \eta}^{v}$ acting on $\mathfrak{S}_{l, \Lambda, \eta}$ by

$$
H_{l, \Lambda, \eta}^{v}=\sum_{\lambda \in Z^{3}(\Lambda, l, \eta)} H_{l, \lambda, \eta}^{v} .
$$

The Hamiltonian (3.12) only includes interactions between particles which are contained in the same cube indexed by $\lambda \in Z^{3}(\Lambda, l, \eta)$.

Suppose the Coulomb Hamiltonian $H_{A}$ on the set $\Lambda$ acts on the Fock space $\mathfrak{S}_{\Lambda}$. For $u \in \Gamma$ we define an injection $i_{u}: \mathfrak{S}_{\Lambda} \rightarrow \mathfrak{S}_{l, \Lambda}, \eta$. Let $\psi\left(x_{1}, \ldots, x_{N}\right)$ be an $N$ particle wave function in $\mathfrak{G}_{\Lambda}$. For $\lambda_{i} \in \mathbf{Z}^{3}, 1 \leqq i \leqq N$, we define a wave function $\psi_{\lambda_{1}, \ldots, \lambda_{N}}$ by

$$
\psi_{\lambda_{1}, \ldots, \lambda_{N}}\left(x_{1}, \ldots, x_{N}\right)=\prod_{i=1}^{N} \chi_{\lambda_{i}}\left(\frac{x_{i}}{l}\right) \psi\left(x_{1}+u l, \ldots, x_{N}+u l\right) .
$$

Then $i_{u} \psi$ is given by

$$
i_{u} \psi=\sum_{\left\{\lambda_{i} \in Z^{3}(\Lambda, l, \eta): 1 \leqq i \leqq N\right\}} \oplus \psi_{\lambda_{1}, \ldots, \lambda_{N}} .
$$

The Hamiltonian induced on $\mathfrak{S}_{\Lambda}$ by $\mathfrak{S}_{l, \Lambda, \eta}^{v}$ is given by $i_{u}^{*} H_{l, \Lambda}^{v}, i_{u}$. We wish next to compare the average of this Hamiltonian for $u \in \Gamma$ to the Coulomb Hamiltonian $H_{A}$.

Lemma 3.1. There is a universal constant $C$ such that if $(v l)^{2} \eta^{5} \geqq C$, then $\left(m \equiv \min m_{i}\right)$

$$
H_{\Lambda}-\int_{\Gamma} i_{u}^{*} H_{l, \Lambda, \eta}^{v} i_{u} d u \geqq-\left[\frac{C N}{m l^{2} \eta}-\frac{1}{2} v \sum_{j=1}^{N} \mathbf{e}_{j}^{2}\right] .
$$

Proof. It is easy to see that the action of the Hamiltonian (3.15) on an $N$ particle wave function is given by [recall identity (2.9)]

$$
-\left[\sum_{i=1}^{N} \frac{1}{2 m_{i}}\right] \frac{1}{l^{2}} \int_{\mathbf{R}^{3}}|\nabla \chi|^{2} d x+\sum_{1 \leqq i<j \leqq N} \mathbf{e}_{i} \mathbf{e}_{j} K_{l}\left(x_{i}-x_{j}\right),
$$


where the function $K_{l}(z)$ is defined by

$$
K_{l}(z)=\frac{1}{l} K^{\nu l}(z / l) \quad \text { and } \quad K^{\mu}(z)=\frac{1}{|z|}-\frac{h(z)}{h(0)} Y_{\mu}(z)
$$

It is clear from the definition of $g(t)$ that

$$
\int_{\mathbf{R}^{3}}|\nabla \chi|^{2} d x \leqq \frac{C}{\eta}
$$

for some universal constant $C$. One sees from (3.17) that $K^{\mu}(0)=\mu$. Hence if we prove that $K^{\mu}(z)$ is a positive definite function then the inequality (3.15) will follow.

To see this we consider the Fourier transform $\hat{K}^{\mu}(p)$ of $K^{\mu}(z)$ given by

$$
\begin{aligned}
\hat{K}^{\mu}(p) & =\frac{4 \pi}{p^{2}}-\frac{1}{(2 \pi)^{3} h(0)} \int_{\mathbf{R}^{3}} \frac{4 \pi}{(p-\xi)^{2}+\mu^{2}} \hat{h}(\xi) d \xi \\
& =\frac{4 \pi \mu^{2}}{p^{2}\left(p^{2}+\mu^{2}\right)}-\frac{1}{(2 \pi)^{3} h(0)} \int\left[\frac{4 \pi}{(p-\xi)^{2}+\mu^{2}}-\frac{4 \pi}{p^{2}+\mu^{2}}\right] \hat{h}(\xi) d \xi \\
& =\frac{4 \pi}{p^{2}+\mu^{2}}\left[\frac{\mu^{2}}{p^{2}}-I(p)+J(p)\right],
\end{aligned}
$$

where

$$
\begin{aligned}
& I(p)=\frac{1}{4 \pi^{3} h(0)} \int \frac{p \cdot \xi \hat{h}(\xi)}{(p-\xi)^{2}+\mu^{2}} d \xi, \\
& J(p)=\frac{1}{8 \pi^{3} h(0)} \int \frac{\xi^{2} \hat{h}(\xi)}{(p-\xi)^{2}+\mu^{2}} d \xi .
\end{aligned}
$$

From (3.8) we see that there is a universal constant $C$ such that

$$
\left[1+|\xi|^{2}\right]^{3}|\widehat{h}(\xi)| \leqq \frac{C}{\eta^{5}}
$$

It follows easily from this that

$$
|J(p)| \leqq C / \eta^{5} p^{2}
$$

for some universal constant $C$. We estimate $I(p)$ by using the fact that $\hat{h}(\xi)=\widehat{h}(-\xi)$, since $h(z)$ is an even function. Thus

$$
\begin{aligned}
I(p) & =\frac{1}{8 \pi^{3} h(0)} \int\left[\frac{1}{(p-\xi)^{2}+\mu^{2}}-\frac{1}{(p+\xi)^{2}+\mu^{2}}\right] p \cdot \xi \hat{h}(\xi) d \xi \\
& =\frac{1}{2 \pi^{3} h(0)} \int \frac{(p \cdot \xi)^{2} \hat{h}(\xi) d \xi}{\left[(p-\xi)^{2}+\mu^{2}\right]\left[(p+\xi)^{2}+\mu^{2}\right]} .
\end{aligned}
$$

It is easy to see now that there is a universal constant $C$ such that

$$
|I(p)| \leqq C / \eta^{5} p^{2}
$$

It follows then from (3.19), (3.23), and (3.25) that if $\mu^{2} \eta^{5} \geqq C$ for some universal constant $C$ then $K^{\mu}(z)$ is positive definite. The inequality (3.15) follows easily now 
from this, (3.18) and the following inequality,

$$
\begin{aligned}
\sum_{1 \leqq i<j \leqq N} \mathbf{e}_{i} \mathbf{e}_{j} K^{\mu}\left(x_{i}-x_{j}\right) & =\frac{1}{2} \int \hat{K}^{\mu}(p)\left[\left|\sum_{j=1}^{N} \mathbf{e}_{j} e^{i p x_{j}}\right|^{2}-\sum_{j=1}^{N} \mathbf{e}_{j}^{2}\right] d p \\
& \geqq-\frac{1}{2} K^{\mu}(0) \sum_{j=1}^{N} \mathbf{e}_{j}^{2} .
\end{aligned}
$$

\section{Bounds on the Global Partition Function}

Here we prove Theorems 1.1 and 1.2 by obtaining upper and lower bounds on the partition function in a large cube $\Lambda$. First we consider the upper bound. From Lemma 3.1 we have the inequality

$$
\begin{aligned}
\beta p_{\Lambda}(\beta, \boldsymbol{\mu}) & =|\Lambda|^{-1} \log \operatorname{Tr} \exp \left[\beta\left(\boldsymbol{\mu} \cdot \mathbf{N}-H_{\Lambda}\right)\right] \\
& \leqq|\Lambda|^{-1} \log \operatorname{Tr} \exp \left[\beta\left(\boldsymbol{\mu}^{\prime} \cdot \mathbf{N}-\int_{\Gamma} i_{u}^{*} H_{l, \Lambda, \eta}^{v} i_{u} d u\right)\right],
\end{aligned}
$$

where $\boldsymbol{\mu}^{\prime}=\left(\mu_{1}^{\prime}, \ldots, \mu_{S}^{\prime}\right)$ and

$$
\mu_{j}^{\prime}=\mu_{j}+\left(C / m l^{2} \eta\right)+v\left(\max z_{\alpha}\right)^{2} .
$$

Now if we use the log convexity of Trace [11] then the term on the right in (4.1) is bounded above by

$$
\begin{aligned}
& \int_{\Gamma} d u|\Lambda|^{-1} \log \operatorname{Tr} \exp \left[\beta\left(\boldsymbol{\mu}^{\prime} \cdot \mathbf{N}-H_{l, \Lambda, \eta}^{v}\right)\right] \\
& \quad \leqq|\Lambda|^{-1} \sum_{\lambda \in Z^{3}(\Lambda, l, \eta)} \log \operatorname{Tr} \exp \left[\beta\left(\boldsymbol{\mu}^{\prime} \cdot \mathbf{N}-H_{l, \lambda, \eta}^{v}\right)\right] \\
& \quad=|\Lambda|^{-1}\left|Z^{3}(\Lambda, l, \eta)\right| \log \operatorname{Tr} \exp \left[\beta\left(\boldsymbol{\mu}^{\prime} \cdot \mathbf{N}-H_{l, 0, \eta}^{v}\right)\right],
\end{aligned}
$$

where $\left|Z^{3}(\Lambda, l, \eta)\right|$ is the number of elements in $Z^{3}(\Lambda, l, \eta)$.

For $\varepsilon>0$ satisfying the condition of Lemma 2.3 we choose $l=e^{5 \varepsilon \beta}$ and $\eta=v$ $=C / l^{2 / 7}$ in such a way that the condition of Lemma 3.1 holds. Then by the remark of Lemma 2.3 we have

$$
\log \operatorname{Tr} \exp \left[\beta\left(\boldsymbol{\mu}^{\prime} \cdot \mathbf{N}-H_{l, 0, \eta}^{v}\right)\right] \leqq \beta\left|U_{\varepsilon}\right| G(\beta, \boldsymbol{\mu})\left[1+O\left(e^{-\varepsilon \beta}\right)\right],
$$

where $U_{\varepsilon}$ is a cube with side of length $l=e^{5 \varepsilon \beta}$. From (3.10) it is easy to see that

$$
\lim _{\Lambda \rightarrow \infty}|\Lambda|^{-1}\left|Z^{3}(\Lambda, l, \eta)\right|\left|U_{\varepsilon}\right|=1 \text {. }
$$

We conclude then from (4.1) to (4.5) that

$$
p(\beta, \mu) \leqq G(\beta, \boldsymbol{\mu})\left[1+O\left(e^{-\varepsilon \beta}\right)\right] .
$$

Since (4.6) is true for all $\mu$ with $\bar{\mu}=O(1 / \beta)$ and $G(\beta, \mu)$ achieves its minimum for some $\bar{\mu}=O(1 / \beta)$, we can replace the right side of (4.6) by $g(\beta, \mu)$ and conclude the proof of the upper bound.

We have proved an upper bound on the pressure. Next we obtain a lower bound. To accomplish this we define the ensemble $G$ on the large cube $\Lambda$. Let $\mathfrak{F}$ be a covering of $A$ with disjoint cubes $U_{\varepsilon}$ with side of length $e^{5 \varepsilon \beta}$. The ensemble $G$ has already been defined on $U_{\varepsilon}$ in Sect. 2. We extend it to $\Lambda$ so that

$$
\Xi_{G}(\beta, \boldsymbol{\mu}, \Lambda)=\prod_{U_{\varepsilon} \in \mathfrak{F}} \Xi_{G}\left(\beta, \boldsymbol{\mu}, U_{\varepsilon}\right) \text {. }
$$


Let $\subseteq$ be the set of all wave functions $\psi$ which are antisymmetric tensor products of the $\psi_{k, i}$ defined in (2.13) with each $\psi_{k, i}$ belonging to a different $U_{\varepsilon} \in \mathfrak{F}$. Then it is clear that

$$
\langle\psi,(H-\boldsymbol{\mu} \cdot \mathbf{N}) \psi\rangle=\sum_{(k, i)}\left\langle\psi_{k, i},(H-\boldsymbol{\mu} \cdot \mathbf{N}) \psi_{k, i}\right\rangle+V_{\mathrm{int}},
$$

where the sum is over the $(k, i)$ such that $\psi_{k, i}$ is included in the tensor product representation for $\psi$. The usual exchange terms vanish because $\psi_{k i}$ and $\psi_{h j}$ have disjoint supports for $k \neq h$. The term $V_{\text {int }}$ is the Coulomb interaction energy between the wave functions $\psi_{k, i}$. Since the wave functions in $\mathbb{S}$ form an orthonormal set, one has that

$$
\begin{aligned}
\operatorname{Tr} \exp \left[\beta\left(\boldsymbol{\mu} \cdot \mathbf{N}-H_{A}\right)\right] & \geqq 1+\sum_{\psi \in \mathcal{E}} \exp [\beta\langle\psi,(\boldsymbol{\mu} \cdot \mathbf{N}-H) \psi\rangle] \\
& =\Xi_{G}(\beta, \boldsymbol{\mu}, \Lambda) E_{G}\left[\exp \left(-\beta V_{\text {int }}\right)\right],
\end{aligned}
$$

where $E_{G}$ denotes the expectation with respect to the ensemble $G$, and we have defined $V_{\text {int }}=0$ on the vacuum. Jensen's inequality yields

$$
E_{G}\left[\exp \left(-\beta V_{\text {int }}\right)\right] \geqq \exp \left[-\beta E_{G}\left(V_{\text {int }}\right)\right] .
$$

From (4.7), (4.9), and (4.10) we obtain then a lower bound on the pressure,

$$
\beta p_{\Lambda}(\beta, \boldsymbol{\mu}) \geqq|\Lambda|^{-1}|\Im| \log \Xi_{G}\left(\beta, \mu, U_{\varepsilon}\right)-\beta|\Lambda|^{-1} E_{G}\left(V_{\text {int }}\right) .
$$

To estimate the interaction term, we first note that the total density of those $\varphi_{i}^{a} s$ corresponding to a fixed eigenvalue $E_{i}(a)$ is radial. This is because the Hamiltonian $H_{\mathrm{N}}^{R, a}$ is rotation invariant. Note that for a particular eigenstate $\varphi_{i}^{a}$, the density corresponding to this state need not be radial! Now we can use Newton's theorem on the $1 / r$ potential (i.e., the mean value property of the harmonic function $1 / r$ ) to write

$$
E_{G}\left(V_{\mathrm{int}}\right)=\frac{1}{2} \sum_{\substack{\mathcal{E}_{\varepsilon}, W_{\varepsilon} \in \mathcal{E}, U_{\varepsilon} \neq W_{\varepsilon}}} I\left(U_{\varepsilon}, W_{\varepsilon}\right)
$$

where

$$
I\left(U_{\varepsilon}, W_{\varepsilon}\right)=\int_{U_{\varepsilon}^{\prime}} \int_{W_{\varepsilon}^{\prime}} \frac{\varrho(x) \varrho(y)}{|x-y|} d x d y .
$$

The cubes $U_{\varepsilon}^{\prime}, W_{\varepsilon}^{\prime}$ are concentric with $U_{\varepsilon}, W_{\varepsilon}$ respectively but with side of length $e^{5 \varepsilon \beta}-3 e^{4 \varepsilon \beta}$. The charge density $\varrho(x)$ is defined for $x \in U_{\varepsilon}^{\prime}$ by

$$
\varrho(x)=\Xi_{G}\left(\beta, \mu, U_{\varepsilon}\right)^{-1} \sum_{k, i}\left|f_{k}(x)\right|^{2} Q^{(i)} \exp \left[-\beta\left\{\frac{\lambda_{k}^{2}}{M^{(i)}}+E_{i}(a)+\bar{\mu} Q^{(i)}\right\}\right] .
$$

Choose $\bar{\mu}$ in such a way that the total charge in a cube is zero, i.e.

$$
\int_{U_{\varepsilon}^{\prime}} \varrho(x) d x=0 .
$$

Clearly, $\bar{\mu}=O(1 / \beta)$ by the same arguments as given in (1.20). We now prove that the contribution from $E_{G}\left(V_{\text {int }}\right)$ is small. First, note that from the symmetry we have

$$
\int_{U_{\varepsilon}^{\prime}} x \varrho(x) d x=0 \text {. }
$$


Now we use Taylor's theorem to expand $|x-y|^{-1}$ and (4.15), (4.16) to estimate $I\left(U_{\varepsilon}, W_{\varepsilon}\right)$. From this we obtain the inequality

$$
\left|I\left(U_{\varepsilon}, W_{\varepsilon}\right)\right| \leqq \frac{27}{4} e^{20 \varepsilon \beta} d\left(U_{\varepsilon}^{\prime}, W_{\varepsilon}^{\prime}\right)^{-5}\left[\int_{U_{\varepsilon}^{\prime}} \varrho \varrho(x) \mid d x\right]^{2},
$$

whence one sees that there is a universal constant $C$ such that

$$
\sum_{W_{\varepsilon}}\left|I\left(U_{\varepsilon}, W_{\varepsilon}\right)\right| \leqq C\left[\int_{U_{\varepsilon}^{\prime}}|\varrho(x)| d x\right]^{2} .
$$

Hence from (4.12) we have that

$$
\left|E_{G}\left(V_{\mathrm{int}}\right)\right| \leqq \frac{C}{2}|\mathscr{F}|\left[\int_{U_{\varepsilon}^{\prime}}|\varrho(x)| d x\right]^{2} .
$$

If we put the inequality (4.19) together with (4.11) and Lemma 2.2 we have then

$$
\beta p_{\Lambda}(\beta, \boldsymbol{\mu}) \geqq|\mathscr{F}|\left|U_{\varepsilon}\right||\Lambda|^{-1}\left[G(\beta, \boldsymbol{\mu})\left[1+O\left(e^{-\varepsilon \beta}\right)\right]-\frac{C}{2} \beta e^{-125 \varepsilon \beta}\left[\int_{U_{\varepsilon}^{\prime}}|\varrho(x)| d x\right]^{2}\right] .
$$

It is clear that if $\varepsilon$ satisfies the condition of Lemma 2.2 then

$$
\left[\int_{U_{\varepsilon}^{\prime}}|\varrho(x)| d x\right]^{2}=G(\beta, \mu) O\left(e^{-\varepsilon \beta}\right) .
$$

Now using the fact that

$$
\lim _{\Lambda \rightarrow \infty}|\mathfrak{F}|\left|U_{\varepsilon}\right||\Lambda|^{-1}=1,
$$

(4.20) and (1.6) yields in the limit $\Lambda \rightarrow \infty$ the lower bound

$$
\beta p(\beta, \mu) \geqq G(\beta, \mu)\left[1+O\left(e^{-\varepsilon \beta}\right)\right] .
$$

Now we can take the infimum on the right side of (4.23) to conclude the lower bound of Theorem 1.1.

Proof of Theorem 1.2. Theorem 1.2 is actually a simple consequence of Theorem 1.1. To see this, first note that $p(\beta, \boldsymbol{\mu})$ is a convex function in $\boldsymbol{\mu}$. Hence the derivative of $p$ can be bounded by finite differences as

$$
\begin{gathered}
-\frac{1}{t}[p(\beta, \boldsymbol{\mu}-t \boldsymbol{\delta})-p(\beta, \boldsymbol{\mu})] \leqq \sum_{\alpha=1}^{S} \frac{\partial p}{\partial \mu_{\alpha}}(\beta, \boldsymbol{\mu})=\varrho(\beta, \boldsymbol{\mu}) \\
\leqq \frac{1}{t}[p(\beta, \boldsymbol{\mu}+t \boldsymbol{\delta})-p(\beta, \boldsymbol{\mu})] \text { for all } t>0,
\end{gathered}
$$

where $\delta=(1, \ldots, 1)$. Because the lower bound is exactly the same as the upper bound, we shall concentrate on the upper bound only. Write $\delta=\delta^{\perp}+\boldsymbol{\delta}^{\prime}$ with $\boldsymbol{\delta}^{\perp} \cdot \mathbf{Z}$ $=\delta^{\perp} \cdot \boldsymbol{\delta}^{\prime}=0$. Now we take $t=O\left(e^{-\varepsilon \beta / 2}\right)$ and use Theorem 1.1 to bound the right side of (4.24) as

$$
\begin{aligned}
p(\beta, \mu) & \leqq \frac{1}{t}\left[g\left(\beta, \mu+t \delta^{\perp}\right)-g(\beta, \mu)\right]+O\left(e^{-\varepsilon \beta / 2}\right) \\
& =\left(\partial g / \partial \boldsymbol{\delta}^{\perp}\right)\left(\beta, \mu^{\prime}\right)+O\left(e^{-\varepsilon \beta / 2}\right),
\end{aligned}
$$


where $\mu^{\prime}$ is some point on the line segment joining $\mu$ and $\mu+t \delta^{\perp}$. Define a function $h(\mu)$ by $h(\mu)=\mu+\omega(\mu) \mathbf{Z}$ with $\omega(\mu)$ satisfying (1.19). By definition

$$
\left(\partial g / \partial \boldsymbol{\delta}^{\perp}\right)\left(\beta, \mu^{\prime}\right)=\left(\partial G / \partial \boldsymbol{\delta}^{\perp}\right)\left(\beta, h\left(\mu^{\prime}\right)\right) .
$$

But $\left(\partial G / \partial \boldsymbol{\delta}^{\prime}\right)\left(\beta, h\left(\mu^{\prime}\right)\right)=0$ (again by definition) and hence

$$
\left(\partial g / \partial \boldsymbol{\delta}^{\perp}\right)\left(\beta, \mu^{\prime}\right)=(\partial G / \partial \boldsymbol{\delta})\left(\beta, h\left(\mu^{\prime}\right)\right)=R\left(\beta, h\left(\mu^{\prime}\right)\right) .
$$

To conclude Theorem 1.2, it remains to show that

$$
R\left(\beta, h\left(\mu^{\prime}\right)\right) \leqq R(\beta, h(\mu))+O\left(e^{-\varepsilon \beta / 2}\right) .
$$

Now by (1.14) we have

$$
\mathbf{e}_{0}(\mu)=\mathbf{e}_{0}\left(\mu^{\prime}\right)+O\left(e^{-\varepsilon \beta / 2}\right),
$$

and hence the relation between $R\left(\beta, h\left(\mu^{\prime}\right)\right)$ and $R(\beta, h(\mu))$ becomes elementary and involves only finite linear combinations of exponential functions. Now (4.27) follows from (4.28) because the exponential functions are differentiable.

\section{Continuity of Stability of Matter Constant}

Here we prove Theorem 1.3 by a modified version of the localization method in Sect. 3.

Let $K$ denote the kinetic energy operator for the Hamiltonian $H^{v}$ and $\varphi(z)$ be the function

$$
\varphi(z)=h(z) /(|z| h(0)),
$$

where $h(z)$ was defined in Sect. 3 and includes a parameter $\eta, 0<\eta<\frac{1}{2}$. For simplicity of notation, we shall assume $m_{\alpha}=\left|z_{\alpha}\right|=1, \alpha=1,2, \ldots, S$.

Write $h(0)=1-2 \delta /(1+\delta)$ [recall from (3.7) that $\delta=O(\eta)]$ and split the Hamiltonian $H^{v}$ into two parts as

$$
H^{v}=\left[\delta K+V_{l}^{\prime}\right]+(1-\delta)\left[K+V_{l}^{\prime \prime}\right] \equiv H^{\prime}+H^{\prime \prime},
$$

where $V_{l}^{\prime \prime}$ is the two body interaction obtained from the potential $\varphi_{l}(z)=l^{-1} \varphi(z / l)$ and $V_{l}^{\prime}$ is defined similarly with the potential $\Phi_{l}$ given by

$$
\Phi_{l}(z)=Y_{v}(z)-(1-\delta) l^{-1} \varphi(z / l) .
$$

The second term in (5.2) can be bounded by the localization method in Sect. 3. We can then use the assumption $H-\mu \cdot \mathbf{N}>\sigma N$ to yield the bound

$$
\begin{aligned}
H^{\prime \prime} & \geqq-\left(C N / l^{2} \eta\right)+(1+\delta)[-\mu \cdot \mathbf{N}+\sigma N] \\
& \geqq-\mu \cdot \mathbf{N}+\sigma N-c_{5}\left(l^{-2} \eta^{-1}+\delta\right) N
\end{aligned}
$$

with $c_{5}=\max \left\{2\left[\max \left|\mu_{\alpha}\right|+\sigma\right], C\right\}$.

Let $d \mu(l)$ be a probability measure on $R^{+}$(which will be fixed later) and define $\Phi_{\mu}$ by

$$
\begin{aligned}
\Phi_{\mu}(z) & =\delta Y_{1}(z)+\left[Y_{v}(z)-\delta Y_{1}(z)-(1-\delta) \int l^{-1} \varphi(z / l) d \mu(l)\right] \\
& \equiv \delta Y_{1}(z)+\Phi_{\mu}^{\prime}
\end{aligned}
$$

with corresponding 2-body interaction $V_{\mu}^{\prime} \equiv \delta V^{(1)}+V_{\mu}^{(2)}$. 
Now integrate the inequality (5.4) with respect to $d \mu(l)$ to obtain

$$
H^{v} \geqq\left[\delta K+\delta V^{(1)}\right]+V_{\mu}^{(2)}+\left[\sigma-c_{5} \delta-c_{5} \eta^{-1} \int l^{-2} d \mu(l)\right]-\mu \cdot \mathbf{N} .
$$

The first bracket can be bounded below by the stability of matter theorem for Yukawa interaction $[2,13]$ as

$$
\delta K+\delta V^{(1)} \geqq-\delta E N,
$$

where $E$ is some universal constant. For the second term $V_{\mu}^{\prime}$, we claim that there is a choice of $d \mu$ such that if

$$
\eta \leqq c^{\prime}|\log v|^{-1 / 6},
$$

then the potential $\Phi_{\mu}^{\prime}$ is positive definite and

$$
\int l^{-2} d \mu(l) \leqq c|\log v|^{-1} .
$$

Here $c$ and $c^{\prime}$ are universal constants. Assuming this, we can then bound $H^{v}$ by

$$
H^{v} \geqq\left\{\sigma-\left[\delta E+\frac{\delta-v}{2}+c_{5} \delta-c c_{5} \eta^{-1}|\log v|^{-1}\right]\right\} N-\mu \cdot \mathbf{N},
$$

where we have used the positivity inequality (3.26) with $\Phi_{\mu}^{\prime}(0)=\delta-v$. Theorem 1.3 is then an immediate consequence of (5.10) and the choice $\delta \sim \eta \sim|\log v|^{-1 / 6}$.

To conclude Theorem 1.3, it remains to prove the existence of such a probability measure.

We choose a probability measure $d \mu(l)$ of the special form

$$
d \mu(l)=\alpha \chi\left(1 \leqq l \leqq v^{-1}\right) l^{-1+\alpha} d l,
$$

where $\chi$ denotes characteristic function and $\alpha$ is given by

$$
\alpha=|\log v|^{-1} \log 2 .
$$

The following two elementary inequalities concerning $d \mu(l)$ will be useful later.

$$
\begin{gathered}
\int \chi(1 \leqq l \leqq A) l^{2} d \mu(l) \leqq \alpha A^{2}, \\
\int \chi\left(A \leqq l \leqq v^{-1}\right) l^{-2} d \mu(l) \leqq \alpha \min \left[1, A^{-2}\right] .
\end{gathered}
$$

Let $\hat{\varphi}(p)$ denote the Fourier transform of $\varphi(z)$. Then from Lemma 3.1 we see that there is a universal constant $c$ such that

$$
\hat{\varphi}(p)-4 \pi / p^{2}=4 \pi A(p) \leqq \begin{cases}c \eta^{-5} p^{-4}, & p>\eta^{-5 / 4} \\ c-4 \pi / p^{2}, & p<\eta^{-5 / 4}\end{cases}
$$

The Fourier transform of $\Phi_{\mu}^{\prime}$ is given by

$$
\frac{1}{4 \pi} \hat{\Phi}_{\mu}^{\prime}=\left(p^{2}+v^{2}\right)^{-1}-\delta\left(p^{2}+1\right)^{-1}-\frac{(1-\delta)}{4 \pi} \int l^{2} \hat{\varphi}(p l) d \mu(l) .
$$

Write $\hat{\Phi}_{\mu}^{\prime}$ as a sum of two terms

$$
\frac{1}{4 \pi} \hat{\Phi}_{\mu}^{\prime}=g(p)-k(p)
$$




$$
\begin{gathered}
g(p)=\left(p^{2}+v^{2}\right)^{-1}-\delta\left(p^{2}+1\right)^{-1}-(1-\delta) p^{-2} \\
=\left[p^{2}\left(\delta-v^{2}\right)-(1-\delta) v^{2}\right]\left[p^{2}\left(p^{2}+1\right)\left(p^{2}+v^{2}\right)\right]^{-1}, \\
k(p)=(1-\delta) \int A(l p) l^{2} d \mu(l) .
\end{gathered}
$$

The last term $k(p)$ can be bounded using (5.13)-(5.16) as

$$
k(p) \leqq k^{\prime}(p)= \begin{cases}c \alpha \nu^{-2}-(1-\delta) p^{-2}, & p \in I \\ c \alpha \eta^{-5} p^{-4}, & p \in I I \\ 2 c \alpha \eta^{-5 / 2} p^{-2}, & p \in I I I\end{cases}
$$

with

$$
I=\left\{p \mid p^{-1} \eta^{-5 / 4} \geqq v^{-1}\right\}, \quad I I=\left\{p \mid p^{-1} \eta^{-5 / 4} \leqq 1\right\},
$$

and

$$
I I I=\left\{p \mid 1 \leqq p^{-1} \eta^{-5 / 4} \leqq v^{-1}\right\} .
$$

We shall assume from now on that

$$
\delta>4 v^{2}
$$

which is certainly satisfied by our choice of $\eta$. The first term $g(p)$ can be bounded by

$$
g(p) \geqq g^{\prime}(p) \equiv \begin{cases}(1-\delta)\left[v^{-2}\left(1+\eta^{-5 / 2}\right)^{-1}-p^{-2}\right], & p \in I \\ \left(\delta / 4 p^{4}\right), & p \in I I \\ \frac{1}{4} \eta^{5 / 2}\left(\delta-v^{2}-\eta^{5 / 2}\right) p^{-2}, & p \in I I I,\end{cases}
$$

where we have used the bounds

$$
\begin{gathered}
\left(p^{2}+v^{2}\right)^{-1} \geqq \delta\left(1+p^{2}\right)^{-1}+(1-\delta)\left(1+\eta^{-5 / 2}\right)^{-1} v^{-2} \quad \text { in } I ; \\
p^{-2}\left(p^{2}+v^{2}\right)^{-1} \geqq \frac{1}{2} p^{-4} \text { and }\left[p^{2}\left(\delta-v^{2}\right)-(1-\delta) v^{2}\right] p^{-2} \geqq \delta / 2 \quad \text { in } I I
\end{gathered}
$$

[recall (5.22) and $\eta<1 / 2]$;

$p^{2}\left(\delta-v^{2}\right)-(1-\delta) v^{2} \geqq p^{2}\left(\delta-v^{2}-\eta^{5 / 2}\right) \quad$ and $\quad p^{2} \geqq\left(p^{2}+1\right)\left(p^{2}+v^{2}\right) \eta^{5 / 2} / 4 \quad$ in $I I I$.

Hence

$$
\begin{gathered}
g(p)-k(p) \geqq 0 \quad \text { if } \quad(1-\delta)>c \alpha\left(1+\eta^{-5 / 2}\right), \quad \delta / 4>c \alpha \eta^{-5}, \\
\delta-v^{2}-\eta^{5 / 2}>2 c \alpha \eta^{-5} .
\end{gathered}
$$

Clearly, $\alpha \sim|\log v|^{-1}$ and $\eta=O\left(|\log v|^{-1 / 6}\right)$ satisfy all these inequalities for small $v$.

\section{Estimates on the Observables}

In this section, we shall present a brief discussions of observables via the "Feynman-Hellman" method. As we shall see, this method applies to a large class of observables once good upper and lower bounds on the partition function are obtained. We shall not define the optimal class of observables which can be estimated by this method. Instead, we shall choose a more specific class of 
observables for which a clean formula can be given. Our class of observables is different from the usual ones (the $n$-point functions) of many-body theory. Our main result is stated in (6.13) and an example is given right after that.

Let $A$ be a multiplication operator defined by

$$
A \psi_{\mathbf{N}}\left(x_{1}, \ldots, x_{N}\right)=A_{\mathbf{N}}\left(x_{1}, \ldots, x_{N}\right) \psi_{\mathbf{N}}\left(x_{1}, \ldots, x_{N}\right) .
$$

By $\log$ convexity we can bound the expectation of $A$ by finite differences as $(t>0)$,

$$
\begin{aligned}
& \frac{1}{t}\left[\log \operatorname{Tr} \exp \left\{\beta\left(\boldsymbol{\mu} \cdot \mathbf{N}-H_{\Lambda}-t A\right)\right\}-\log \operatorname{Tr} \exp \left\{\beta\left(\boldsymbol{\mu} \cdot \mathbf{N}-H_{A}\right)\right\}\right] \\
& \quad \leqq\langle A\rangle \leqq \frac{1}{t}\left[\log \operatorname{Tr} \exp \left\{\beta\left(\boldsymbol{\mu} \cdot \mathbf{N}-H_{\Lambda}+t A\right)\right\}-\log \left\{\beta\left(\boldsymbol{\mu} \cdot \mathbf{N}-H_{A}\right)\right\}\right] .
\end{aligned}
$$

To apply (6.2), one should have good lower and upper bounds on the pressure in the presence of small perturbations $t A$. Let us further assume that $A_{\mathrm{N}}$ is given by

$$
A_{\mathrm{N}}\left(x_{1}, \ldots, x_{N}\right)=\int_{\Lambda} d u f_{N}\left(x_{1}-u, \ldots, x_{N}-u\right) .
$$

The functions $f_{N}$ are defined in terms of symmetric functions $g_{k}$ of $k$ variables $y_{1}, \ldots, y_{k}, k=1,2, \ldots$, which are supported in the set $\left|y_{i}\right|<\zeta, i=1, \ldots, k$. To define $f_{N}\left(y_{1}, \ldots, y_{N}\right)$ let $\xi>0$ and suppose the set of $y_{i}, 1 \leqq i \leqq N$, such that $\left|y_{i}\right|<\xi$ is given by $y_{i}, i=1,2, \ldots, \theta(\xi)$, and the center of mass of these $y_{i}$ is $\bar{y}(\xi)$. Then we have

$$
f_{N}\left(y_{1}, \ldots, y_{N}\right)= \begin{cases}0 & \text { if } \theta(\xi)=0 \\ \frac{3}{4 \pi \xi^{3}} g_{\theta(\xi)}\left(y_{1}-\bar{y}(\xi), \ldots, y_{\theta(\xi)}-\bar{y}(\xi)\right) & \text { if } \theta(\xi)>0 .\end{cases}
$$

Thus in the definition of $f_{N}$ there are two parameters $\xi, \zeta$, involved as well as the functions $g_{k}$. These parameters are chosen fixed, depending on $\beta$, with

$$
\xi=O\left(e^{\varepsilon \beta}\right), \quad \zeta=O(\beta) .
$$

We shall also assume that the $g_{k}$ can be bounded,

$$
\left\|g_{k}\right\|_{\infty}<c k
$$

for some universal constant $c$. The assumption (6.6) is important since otherwise $t A$ can not be viewed as a small perturbation of $H$ and the physics described by $H+t A$ can be completely different from that described by $H$.

With this assumption, it is not difficult to check that the localization method in Sect. 3 can be extended to the operator $H \pm t A$ with the localized Hamiltonian $H_{l, \lambda, \eta}^{v}$ replaced by $H_{l, \lambda, \eta}^{v} \pm t A_{l, \lambda}$. Here the operator $A_{l, \lambda}$ is given by

$$
A_{l, \lambda} \psi\left(x_{1}, \ldots, x_{N}\right)=\int_{Q} d u f_{N}\left(x_{1}-u, \ldots, x_{N}-u\right) \psi\left(x_{1}, \ldots, x_{N}\right),
$$

where $Q$ is the set of $u$ which are within a distance $\xi$ from the cube with center $l \lambda$ and side of length $l(1+2 \eta)$. Equation (6.7) reduces the estimate of the pressure to that of a cube with size of order $l$ and Yukawa interactions. For this purpose, our first task is to estimate the shifts of the ground state energy under small perturbations. A natural tool for this is to make standard second order perturbation theory rigorous. In the case of the ground state energy with a gap, this is not difficult to 
achieve by combining usual perturbation arguments with the variational principle.

To avoid discussion of degenerate perturbation theory, we shall assume that the ground states $\psi_{1}, \ldots, \psi_{g}$ of $H^{R}-\mu \cdot \mathbf{N}$ have different particle numbers, namely $\mathbf{N}^{(i)} \neq \mathbf{N}^{(j)}$ if $i \neq j\left[\right.$ the definition of $\mathbf{N}^{(i)}$ is given in Sect. 1 before assumption $\left.(A)\right]$. The general case can be treated by degenerate perturbation theory but the notation becomes too clumsy and will not be discussed here. With this assumption, Lemma 2.1 can be easily modified using standard perturbation theory. We leave the details to the reader and simply write down the following conclusion:

$$
\left|\mathbf{e}_{j}^{v}(\mu, t A)-\left(\mathbf{e}_{0}(\mu)+t w_{j}\right)\right|<\tau^{-1} O\left(t^{2}\right)+O\left(l^{-2}\right),
$$

where $\tau$ is the gap defined in the corollary of Theorem 1.3 and $w_{j}$ is defined by

$$
w_{j}=\int\left|\psi_{j}\left(x_{1}, \ldots, x_{N^{(j)}}\right)\right|^{2} g_{N^{(j)}}\left(x_{1}, \ldots, x_{N^{(j)}}\right) d \bar{x},
$$

with $d \bar{x}$ denoting the standard Lebesgue measure restricted to the subspace $\sum m_{i} x_{i}=0$. Note that we need a universal bound $\left\|g_{N}\right\|_{\infty}<C$ for all $N<N_{0}$ to make use of second order perturbation theory. This bound is again provided by (6.6). Now we can apply the method of Lemma 2.3 to conclude an upper bound for the pressure in a cube. By the above remarks on localization, this bound extends to the pressure on the whole box $\Lambda$. Hence we have the following bound for $t=O\left(e^{-\varepsilon \beta / 2}\right)$,

$$
\beta p(\beta, \mu, t A) \leqq \sum_{i=1}^{g} \varrho^{(i)} \exp \left[-\beta t w_{i}\right]\left(1+O\left(e^{-\varepsilon \beta / 2}\right)\right) .
$$

Here the function $\varrho^{(i)}$ is the "reduced density" of the $i^{\text {th }}$-complex defined by [cf. (1.17)]

$$
\varrho^{(i)}=\left(\frac{M^{(i)}}{2 \pi \beta}\right)^{3 / 2} \exp \left[-\beta \mathbf{e}_{i}(\boldsymbol{\mu})\right]
$$

with $\boldsymbol{\mu}=\mu+\bar{\mu} \mathbf{Z}$ and $\bar{\mu}$ solves (1.19).

A lower bound to the pressure similar to $(6.10)$ is much easier to obtain since it is just a variational calculation. Putting this lower bound together with the upper bound (6.10), we have succeeded in extending Theorem 1.1 to the case with small perturbations. To summarize, for $A$ satisfying (6.5) and (6.6) the pressure can be bounded (for $t \sim e^{-\varepsilon \beta / 2}$ ) by

$$
\beta p(\beta, \mu, t A)=\sum_{i=1}^{g} \varrho^{(i)} \exp \left[-\beta t w_{i}\right]\left(1+O\left(e^{-\varepsilon \beta / 2}\right)\right) .
$$

We can now use (6.12) in (6.2) to bound $\langle A\rangle$ by

$$
|\Lambda|^{-1}\langle\mathrm{~A}\rangle=\sum_{i=1}^{g}\left[\varrho^{(i)} w_{i}\left(1+O\left(e^{-\varepsilon \beta / 2}\right)\right)+\varrho^{(i)} O\left(e^{-\varepsilon \beta / 2}\right)\right] .
$$

In case $w_{i} \neq 0$ the second term in the bracket can be absorbed into the $O\left(e^{-\varepsilon \beta / 2}\right)$ part of the first term, while for $w_{i}=0$ the first term vanishes and only the second term is left. Equation (6.13) is the main result in this section. It shows that the expectation value of an observable is given by the expectation value of that observable in the ideal gas of ground state atoms or molecules plus a fluctuation of order $e^{-\varepsilon \beta / 2}$. 
Let us specialize (6.13) to the following example to have some perspective and to compare with previous results for the hydrogen atom [5].

Let $g_{k}$ be the local number operator defined by

$$
g_{k}\left(y_{1}, \ldots, y_{k}\right)=\sum_{i=1}^{k} \chi_{\zeta}\left(y_{i}\right)
$$

where $\chi_{\zeta}$ denotes the characteristic function of the set $|y|<\zeta$. The corresponding operator $A$ can be interpreted as the local density operator. If one takes into account that $\psi_{j}$ has exponential decay (see [9] for a discussion and references) the expectation $w_{j}$ can then be bounded by

$$
\left|w_{j}-N^{(j)}\right|<O\left(e^{-\varepsilon \beta}\right) .
$$

Hence Eq. (6.13) asserts that the local density is indeed the same as the global density (given by Theorem 1.2) with accuracy $O\left(e^{-\varepsilon \beta / 2}\right)$. Note that this is an assertion concerning the density fluctuations. In particular, if we let $g_{k}=0$ in (6.14) for $k \in\left\{N^{(i)} \mid i=1, \ldots, g\right\}, A$ can be interpreted as the local density for "complexes" other than the ground state complexes. In this case, (6.13) shows that the expected density is then smaller by a factor of order $e^{-\varepsilon \beta / 2}$, which is a stronger statement than the corresponding statement for the hydrogen atom which appeared in [5].

\section{Appendix}

Here we show how our localization method can be applied to prove a result similar to but stronger than Lemma 2.1. The advantage of this result over the simpler proof of Lemma 2.1 is that the constant involved depends only on the ground state eigenvalue of the Hamiltonian and not on the ground state eigenfunctions.

Lemma A.1. With the same assumptions as Lemma 2.1 there is the inequality

$$
\mathbf{e}_{0}(\boldsymbol{\mu}) \leqq E_{i}(a) \leqq \mathbf{e}_{0}(\boldsymbol{\mu})+C / a^{2}, \quad a \geqq 1,
$$

where the constant $C$ depends only on $\mathbf{e}_{0}(\boldsymbol{\mu})$.

Proof. For simplicity of notation we shall take $\boldsymbol{\mu}=\left(\mu_{1}, \ldots, \mu_{\mathrm{S}}\right)$ to have all components equal, $\mu_{i}=\mu$, whence $\boldsymbol{\mu} \cdot \mathbf{N}=\mu N, \mathbf{e}_{0}(\boldsymbol{\mu})=\mathbf{e}_{0}(\mu)$.

The lower bound on $E_{i}(a)$ in (A.1) follows easily from the Rayleigh-Ritz principle so we shall concentrate on the upper bound. The main tool we shall use is Lemma 3.1. Let us fix a particular eigenspace of $\mathbf{N}$, namely $\mathbf{N}=\mathbf{N}_{0}$, and assume that $H^{R}-\mu N$ has ground state energy $\mathbf{e}_{0}(\mu)$ on this eigenspace with degeneracy $g \geqq 1$. The Hamiltonian $H^{R}$ is given by the formula (1.2) with $\mathbf{N}=\mathbf{N}_{0}$ and acts on wave functions $\psi\left(x_{1}, \ldots, x_{N}\right)$ on $\mathbf{R}^{3 N}$ which are constant along 3 dimensional hyperplanes orthogonal to the subspace

$$
\begin{gathered}
\sum_{i=1}^{N} m_{i} x_{i}=0, \quad \text { with respect to the quadratic form } \\
\sum_{i=1}^{N} m_{i}\left|x_{i}\right|^{2} .
\end{gathered}
$$

The norm of $\psi,\|\psi\|$ is the $L^{2}$ norm of $\psi$ restricted to the subspace (A.2) which is induced by the quadratic form (A.3). We denote this Hilbert space by $\mathfrak{G}_{N}^{R}$. 
Let $\lambda=\left(\lambda_{1}, \lambda_{2}, \ldots, \lambda_{N}\right) \in \mathbf{Z}^{3 N}$ denote a multi index and $\psi_{\lambda, u}$ be defined for $u \in \Gamma$ by

$$
\psi_{\lambda, u}\left(x_{1}, \ldots, x_{N}\right)=\prod_{i=1}^{N} \chi_{\lambda_{i}}\left(\frac{x_{i}}{l}+u\right) \psi\left(x_{1}, \ldots, x_{N}\right)
$$

The Hamiltonian $H_{\lambda}^{v}$ is given by

$$
H_{\lambda}^{v}=-\sum_{i=1}^{N} \frac{1}{2 m_{i}} \Delta_{i}+\frac{1}{h(0)} \sum_{1 \leqq i<j \leqq N} \delta_{\lambda_{i} \lambda_{j}} \mathbf{e}_{i} \mathbf{e}_{j} Y_{v}\left(x_{i}-x_{j}\right)
$$

and is assumed to act on functions in $\mathfrak{H}_{N}^{R}$. Then if we let $P \psi_{\lambda, u} \in \mathfrak{H}_{N}^{R}$ be defined by $P \psi_{\lambda, u}=\psi_{\lambda, u}$ on the subspace (A.2) it is clear from Lemma 3.1 that

$$
\left\langle\psi,\left(H^{R}-\mu N\right) \psi\right\rangle \geqq \int_{\Gamma} d u \sum_{\lambda \in \mathbf{Z}^{3 N}}\left\langle P \psi_{\lambda, u}\left(H_{\lambda}^{v}-\mu N\right) P \psi_{\lambda, u}\right\rangle-\left[\frac{C}{m l^{2} \eta}+v\right] N .
$$

For $\alpha \in \mathbf{Z}^{3}, \lambda \in \mathbf{Z}^{3 N}$ let $N_{\lambda}(\alpha)$ be

$$
N_{\lambda}(\alpha)=\#\left\{\lambda_{i}: \lambda_{i}=\alpha, 1 \leqq i \leqq N\right\} .
$$

Then by the Rayleigh-Ritz principle and scaling one has

$$
H_{\lambda}^{v}-\mu N \geqq \sum_{\left\{\alpha \in \mathbf{Z}^{3}: N_{\lambda}(\alpha) \neq 0\right\}} \frac{\mathbf{e}_{0}(\mu)+\mu N_{\lambda}(\alpha)}{h(0)^{2}}-\mu N_{\lambda}(\alpha)-\frac{v\left[N_{\lambda}(\alpha)-1\right] N_{\lambda}(\alpha)}{2 h(0)} .
$$

Now let $\psi_{i}, i=1,2, \ldots, g$ be the ground state wave functions in $\mathfrak{S}_{N}^{R}$ of $H^{R}-\mu N$ with energy $\mathbf{e}_{0}(\mu)$. Putting $\psi=\psi_{i}, i=1, \ldots, g$ in (A.6) and summing we obtain the inequality

$$
\mathbf{g e}_{0}(\mu) \geqq \int_{\Gamma} F(u) d u-\left[\frac{C}{m l^{2} \eta}+v\right] g N .
$$

From (A.8) we obtain a lower bound on $F(u)$,

$$
F(u) \geqq g\left\{[1+O(\eta)] \mathbf{e}_{0}(\mu)+\mu N O(\eta)-v N^{2}\right\} .
$$

Hence if we put $G(u)=F(u)-g \mathbf{e}_{0}(\mu)$, then (A.9) and (A.10) yield the inequalities

$$
\begin{gathered}
G(u) \geqq O(\eta) \mathbf{e}_{0}(\mu)+\mu N O(\eta)-v N^{2}, \\
\int_{\Gamma} G(u) d u \leqq\left[\frac{C}{m l^{2} \eta}+v\right] g N .
\end{gathered}
$$

Let $\Gamma_{0} \subset \Gamma$ be a cube with center at the origin and side of length $L_{0}<1$. Then it is clear from (A.11) and (A.12) that there is a $u_{0} \in \Gamma_{0}$ such that

$$
G\left(u_{0}\right) \leqq L_{0}^{-3}\left\{\left[\frac{C}{m l^{2} \eta}+v\right] g N-O(\eta) \mathbf{e}_{0}(\mu)-\mu N O(\eta)+v N^{2}\right\} .
$$

For $a \geqq 1$ let $E_{\mathbf{N}, i}(a), i=1,2, \ldots, g$ be the first $g$ eigenvalues of $H^{R, a}-\mu N$ on the eigenspace of $\mathbf{N}$. We choose $a$ such that

$$
a=\frac{\sqrt{3}}{2}\left[1+L_{0}\right] l[1+O(\eta)] .
$$


Then by Rayleigh-Ritz and scaling it follows that if $\lambda=0 \in \mathbf{Z}^{3 N}$ then

$$
\sum_{i=1}^{g} E_{\mathrm{N}, i}(a) \leqq \kappa[1+O(\eta)] \sum_{i=1}^{g}\left[\left\langle P \psi_{i, 0, u_{0}}\left(H_{0}^{\nu}-\mu N\right) P \psi_{i, 0, u_{0}}\right\rangle+\frac{v N^{2}}{2}+O(\eta) \mu N\right],
$$

where $\kappa$ is defined by

$$
\kappa^{-1}=\min _{1 \leqq i \leqq g}\left\{a_{i i}-\frac{1}{2} \sum_{j \neq i}\left|a_{i j}\right|+\left|a_{j i}\right|\right\},
$$

and $A=\left(a_{i j}\right)$ is the matrix determined by the transformation

$$
P \psi_{i, 0, u_{0}}=\sum_{j=1}^{g} a_{i j} v_{j}
$$

where $v_{i}, 1 \leqq i \leqq g$ is an orthonormal set.

Next we take $L_{0}=\frac{1}{4}, \eta=v$, and $l^{2} \eta^{7}=C$ in such a way that the condition of Lemma 3.1 is satisfied. It follows then from (A.15) and (A.13) that if $\kappa \leqq 1+C a^{-2 / 7}$ then

$$
\sum_{i=1}^{g} E_{\mathbf{N}, i}(a) \leqq g \mathbf{e}_{0}(\mu)+\frac{C N^{2}}{a^{2 / 7}} .
$$

Since we already know that $E_{\mathbf{N}, i}(a) \geqq \mathbf{e}_{0}(\mu), 1 \leqq i \leqq g$ Lemma A.1 follows provided we prove the bound on $\kappa$.

To accomplish this let $L$ be the $g \times g$ matrix

$$
L_{i j}=\left\langle P \psi_{i, 0, u_{0}}, P \psi_{j, 0, u_{0}}\right\rangle \text {. }
$$

Then if $L$ is nonsingular $A=L^{1 / 2}$. We shall show that

$$
\|L-I\| \leqq \frac{C}{a^{2 / 7}}
$$

whence the bound on $\kappa$ follows. To see this note that if $\lambda \in \mathbf{Z}^{3 N}$ has all $\lambda_{i}$ equal then $P \psi_{\lambda, u_{0}} \equiv 0$ unless $\lambda=0$. Also by (A.8) if $\lambda$ is such that not all $\lambda_{i}$ are equal then

$$
H_{\lambda}^{v}-\mu N \geqq 2 \mathbf{e}_{0}(\mu)+C a^{-2 / 7} .
$$

It follows then from (A.13) and (A.21) that

$$
\left\|\psi_{i}-P \psi_{i, 0, u_{0}}\right\|^{2} \leqq C a^{-2 / 7}, \quad 1 \leqq i \leqq g
$$

Since the $\psi_{i}$ form an orthonormal set the inequality (A.20) follows. We can now argue as in Lemma 2.1 to obtain the inequality A.1.

Acknowledgements. The authors would like to thank C. Fefferman, G. W. Ford, and D. Brydges for helpful comments.

\section{References}

1. Brydges, D., Federbush, P.: The cluster expansion for potentials with exponential fall-off. Commun. Math. Phys. 53, 19-30 (1977)

2. Conlon, J.G., Lieb, E.H., Yau, H.-T.: The $N^{7 / 5}$ Law for charged bosons. Commun. Math. Phys. 116, 417-448 (1988) 
3. Cycon, H.L., Froese, R.G., Kirsch, W., Simon, B.: Schrödinger operators. Berlin, Heidelberg, New York: Springer 1987

4. Dyson, F.J., Lenard, A.: Stability of matter. I, II, J. Math. Phys. 8, $423-434$ (1967); ibid 9, 698-711 (1968)

5. Fefferman, C.: The atomic and molecular nature of matter. Rev. Math. Iberoamericana 1, 1-44 (1985)

6. Lieb, E.H.: The stability of matter. Rev. Mod. Phys. 48, 553-569 (1976)

7. Lebowitz, J.L., Pena, R.E.: Low density form of the free energy for real matter. J. Chem. Phys. 59, 1362-1364 (1973)

8. Lieb, E.H., Lebowitz, J.L.: The constitution of matter: existence of thermodynamics for systems composed of electrons and nuclei. Adv. Math. 9, 316-398 (1972)

9. Reed, M., Simon, B.: Methods of modern mathematical physics. IV. analysis of operators. New York: Academic Press 1978

10. Richards, P.I.: Manual of mathematical physics. New York: Pergamon Press 1959. This is a useful reference for a succinct presentation of the Saha equation in a general setting

11. Ruelle, D.: Statistical mechanics rigorous results. Reading, MA: Benjamin 1969

12. Saha, M.N.: Ionization in the solar chromosphere. Phil. Mag. 40, 472-488 (1920)

13. Thirring, W.E.: A course in mathematical physics, Vol. 4. Quantum mechanics of large systems. Wien, New York: Springer 1983

Communicated by A. Jaffe

Received March 21, 1989 